\title{
Characterizing Derivations on Von Neumann Algebras by Local Actions
}

\author{
Xiaofei Qi and Jia Ji \\ Department of Mathematics, Shanxi University, Taiyuan 030006, China \\ Correspondence should be addressed to Xiaofei Qi; xiaofeiqisxu@aliyun.com
}

Received 25 August 2013; Accepted 25 November 2013

Academic Editor: P. Veeramani

Copyright (C) 2013 X. Qi and J. Ji. This is an open access article distributed under the Creative Commons Attribution License, which permits unrestricted use, distribution, and reproduction in any medium, provided the original work is properly cited.

Let $\mathscr{M}$ be any von Neumann algebra without central summands of type $I_{1}$ and $P$ a core-free projection with the central carrier $I$. For any scalar $\xi$, it is shown that every additive map $L$ on $\mathscr{M}$ satisfies $L(A B-\xi B A)=L(A) B-\xi B L(A)+A L(B)-\xi L(B) A$ whenever $A B=P$ if and only if (1) $\xi=1, L=\varphi+h$, where $\varphi$ is an additive derivation and $h$ is a central valued additive map vanishing on $A B-B A$ with $A B=P ;(2) \xi \neq 1, L$ is a derivation with $L(\xi A)=\xi L(A)$ for each $A \in \mathscr{M}$

\section{Introduction}

Let $\mathscr{A}$ be an algebra over a field $\mathbb{F}$. For a scalar $\xi \in \mathbb{F}$ and for $A, B \in \mathscr{A}$, we say that $A$ commutes with $B$ up to a factor $\xi$ if $A B=\xi B A$. The notion of commutativity up to a factor for pairs of operators is an important concept and has been studied in the context of operator algebras and quantum groups $[1,2]$. Motivated by this, a binary operation $[A, B]_{\xi}=$ $A B-\xi B A$, called $\xi$-Lie product of $A$ and $B$, was introduced in [3]. An additive map $L: \mathscr{A} \rightarrow \mathscr{A}$ is called an additive $\xi$ Lie derivation if $L\left([A, B]_{\xi}\right)=[L(A), B]_{\xi}+[A, L(B)]_{\xi}$ holds for all $A, B \in \mathscr{A}$. It is clear that a $\xi$-Lie derivation is a derivation if $\xi=0$; is a Lie derivation if $\xi=1$; is a Jordan derivation if $\xi=-1$. The structure of $\xi$-Lie derivations on various operator algebras was discussed by several authors (see [3-5] and the references therein).

Recently, the question of under what conditions that a map becomes a (Jordan) derivation attracted much attention of many researchers (see [6-9] and the references therein). For $\xi$-Lie derivations, an additive (a linear) map $L$ on $\mathscr{A}$ is said to be $\xi$-Lie derivable at a point $Z \in \mathscr{A}$ if $L\left([A, B]_{\xi}\right)=$ $[L(A), B]_{\xi}+[A, L(B)]_{\xi}$ for any $A, B \in \mathscr{A}$ with $[A, B]_{\xi}=Z$. Clearly, this definition is only valid for $\xi$-Lie commutators, that is, the elements of the form $Z=[A, B]_{\xi}$. For instance, if $Z=I$ and $\xi=1$, as the unit $I$ may not be a commutator in general, there is no sense to define that $L$ is Lie derivable at $I$. Since zero is a $\xi$-Lie commutator for any $\xi$ and any algebra, as a start, Qi and Hou [10] characterized the linear maps Lie derivable at zero between $\mathscr{J}$-subspace lattice algebras. In [11] Qi et al. considered further the additive maps $\xi$ Lie derivable at zero on unital prime algebras over a field $\mathbb{F}$ containing a non-trivial idempotent $P$. Since factor von Neumann algebras are prime, as a consequence of the result for prime algebras, all additive maps $\xi$-Lie derivable at zero on factor von Neumann algebras are characterized. Recently, Qi et al. [12] gave a characterization of additive maps $\xi$-Lie derivable at zero on general von Neumann algebras for all possible $\xi$.

Lu and Jing [13] considered the question of characterizing Lie derivations from another direction. Let $X$ be a Banach space with $\operatorname{dim} X \geq 3$ and $\mathscr{B}(X)$ the algebra of all bounded linear operators acting on $X$. They [13] showed that, if $\delta$ : $\mathscr{B}(X) \rightarrow \mathscr{B}(X)$ is a linear map satisfying $\delta([A, B])=$ $[\delta(A), B]+[A, \delta(B)]$ for any $A, B \in \mathscr{B}(X)$ with $A B=0$ (resp., $A B=P$, where $P$ is a fixed nontrivial idempotent), then $\delta=\tau+\nu$, where $\tau$ is a derivation of $\mathscr{B}(X)$ and $v$ : $\mathscr{B}(X) \rightarrow \mathbb{C} I$ is a linear map vanishing at commutators $[A, B]$ with $A B=0$ (resp., $A B=P$ ). Later, this result was generalized to the additive maps on triangular algebras and prime rings in $[14,15]$, respectively. Let $\mathscr{M}$ be a von Neumann algebra without central summands of type $I_{1}$ and $L: \mathscr{M} \rightarrow \mathscr{M}$ an additive map. For $\xi$-Lie derivations, Qi and Hou [16] showed that $L$ satisfies $L\left([A, B]_{\xi}\right)=[L(A), B]_{\xi}+[A, L(B)]_{\xi}$ for any $A, B$ with $A B=0$ if and only if there exists an additive 
derivation $\varphi$ such that (1) $\xi=1, L=\varphi+f$, where $f$ : $\mathscr{M} \rightarrow \mathscr{Z}(\mathscr{M})$ is an additive map vanishing each commutator $[A, B]$ whenever $A B=0$; (2) $\xi=-1, L=\varphi$; (3) $\xi=0$, $L(A)=\varphi(A)+L(I) A$ for all $A ;(4) \xi \notin\{0, \pm 1\}, \varphi(\xi A)=\xi L(A)$, and $L(A)=\varphi(A)+L(I) A$ for all $A$.

Let $\mathscr{M}$ be any von Neumann algebra and let $A \in \mathscr{M}$. Recall that the central carrier of $A$, denoted by $\bar{A}$, is the intersection of all central projections $P$ such that $P A=0$. If $A$ is self-adjoint, then the core of $A$, denoted by $A$, is $\sup \left\{S \in \mathscr{Z}(\mathscr{M}): S=S^{*}, S \leq A\right\}$. Particularly, if $A=P$ is a projection, it is clear that $\underline{P}$ is the largest central projection $\leq P$. A projection $P$ is called core free if $\underline{P}=0$. It is easy to see that $\underline{P}=0$ if and only if $\overline{I-P}=I$. By [17], if $\mathscr{M}$ is a von Neumann algebra without central summands of type $I_{1}$, there exists a nonzero core-free projection $P \in \mathscr{M}$ with $\bar{P}=I$. So, it is easily seen that $\mathscr{M}$ is a von Neumann algebra without central summands of type $I_{1}$ if and only if it has a projection $P$ with $\underline{P}=0$ and $\bar{P}=I$. Fixed such $P$. The purpose of the present paper is to give a complete characterization of additive maps $L$ satisfying $L\left([A, B]_{\xi}\right)=[L(A), B]_{\xi}+[A, L(B)]_{\xi}$ for any $A, B$ with $A B=P$ on von Neumann algebras without central summands of type $I_{1}$ for all possible $\xi$.

Let $\mathscr{M}$ be a von Neumann algebra without central summands of type $I_{1}$ and $P \in \mathscr{M}$ a nonzero core-free projection with $\bar{P}=I$. Assume that $L: \mathscr{M} \rightarrow \mathscr{M}$ is an additive map. In this paper, we prove that $L$ satisfies $[L(A), B]_{\xi}+[A, L(B)]_{\xi}=$ $L\left([A, B]_{\xi}\right)$ for any $A, B \in \mathscr{M}$ with $A B=P$ if and only if (1) $\xi=1, L(A)=\varphi(A)+h(A)$ for all $A \in \mathscr{M}$, where $\varphi: M \rightarrow M$ is an additive derivation, $h: \mathscr{M} \rightarrow \mathscr{Z}(\mathscr{M})$ is an additive map vanishing each commutator $[A, B]$ whenever $A B=P$, and $\mathscr{Z}(\mathscr{M})$ denotes the center of $\mathscr{M}$ (Theorem 1$)$; (2) $\xi \neq 1, L$ is an additive derivation satisfying $L(\xi A)=\xi L(A)$ for all $A$ (Theorem 4).

\section{Characterizing Lie Derivations by Local Action}

In this section, we consider the question of characterizing Lie derivations by local action at a core-free projection $P$ with $\bar{P}=I$ on general von Neumann algebras having no central summands of type $I_{1}$.

Theorem 1. Let $M$ be a von Neumann algebra without central summands of type $I_{1}$ and $P \in \mathscr{M}$ a nonzero core-free projection with $\bar{P}=I$. Suppose that $L: \mathscr{M} \rightarrow \mathscr{M}$ is an additive map. Then $L$ satisfies $L([A, B])=[L(A), B]+[A, L(B)]$ for any $A, B \in$ $\mathscr{M}$ with $A B=P$ if and only if there exists an additive derivation $\varphi: \mathscr{M} \rightarrow \mathscr{M}$ and an additive map $h: \mathscr{M} \rightarrow \mathscr{Z}(\mathscr{M})$ vanishing each commutator $[A, B]$ whenever $A B=P$ such that $L(A)=\varphi(A)+h(A)$ for all $A \in \mathscr{M}$, where $\mathscr{Z}(\mathscr{M})$ denotes the center of $\mathscr{M}$.

We first give two lemmas, which are needed.

Lemma 2 (see [17]). Let $\mathscr{M}$ be a von Neumann algebra. For projections $P, Q \in \mathscr{M}$, if $\bar{P}=\bar{Q} \neq 0$ and $P+Q=I$, then $T \in \mathscr{M}$ commutes with PXQ and QXP for all $X \in \mathscr{M}$ implies $T \in$ $\mathscr{Z}(\mathscr{M})$.
Lemma 3 (see [16]). Let $M$ be a von Neumann algebra. Assume that $P \in \mathscr{M}$ is a projection with $\underline{P}=0$ and $\bar{P}=I$. Then $P \mathscr{M} P \cap \mathscr{Z}(\mathscr{M})=(I-P) \mathscr{M}(I-P) \cap \mathscr{Z}(\mathscr{M})=\{0\}$.

Proof of Theorem 1. By the definitions of core and central carrier, $I-P$ is also core free and $\overline{I-P}=I$. For convenience, denote $\mathscr{M}_{i j}=P_{i} \mathscr{M} P_{j}, i, j \in\{1,2\}$, where $P_{1}=P$ and $P_{2}=$ $I-P$. Then $\mathscr{M}=\mathscr{M}_{11}+\mathscr{M}_{12}+\mathscr{M}_{21}+\mathscr{M}_{22}$.

The "if" part is obvious. We will prove the "only if" part by checking several claims.

Claim 1. Consider $P_{1} L\left(P_{1}\right) P_{1}+P_{2} L\left(P_{1}\right) P_{2} \in \mathscr{Z}(\mathscr{M})$. Since $I P_{1}=P_{1}$, we have $0=L\left(\left[I, P_{1}\right]\right)=\left[L(I), P_{1}\right]+\left[I, L\left(P_{1}\right)\right]$, and so

$$
L(I) P_{1}=P_{1} L(I)
$$

For any $A_{12} \in \mathscr{M}_{12}$, by $\left(I+A_{12}\right) P_{1}=P_{1}$, we get $L([I+$ $\left.\left.A_{12}, P_{1}\right]\right)=\left[L(I)+L\left(A_{12}\right), P_{1}\right]+\left[I+A_{12}, L\left(P_{1}\right)\right]$; that is,

$$
\begin{aligned}
& L\left(A_{12}\right)+L(I) P_{1}+L\left(A_{12}\right) P_{1}-P_{1} L(I) \\
& \quad-P_{1} L\left(A_{12}\right)+A_{12} L\left(P_{1}\right)-L\left(P_{1}\right) A_{12}=0 .
\end{aligned}
$$

Let $L\left(A_{12}\right)=S_{11}+S_{12}+S_{21}+S_{22}$. Combining (2) with (1), one gets

$$
\begin{aligned}
0= & S_{11}+A_{12} L\left(P_{1}\right) P_{1}+A_{12} P_{2} L\left(P_{1}\right) P_{2} \\
& -P_{1} L\left(P_{1}\right) P_{1} A_{12}+2 S_{21}+S_{22}-P_{2} L\left(P_{1}\right) P_{1} A_{12} .
\end{aligned}
$$

It follows that $A_{12} P_{2} L\left(P_{1}\right) P_{2}=P_{1} L\left(P_{1}\right) P_{1} A_{12}$, which implies

$$
\begin{aligned}
P_{1} A P_{2} & \left(P_{2} L\left(P_{1}\right) P_{2}+P_{1} L\left(P_{1}\right) P_{1}\right) \\
= & \left(P_{2} L\left(P_{1}\right) P_{2}+P_{1} L\left(P_{1}\right) P_{1}\right) P_{1} A P_{2}
\end{aligned}
$$

for all $A \in \mathscr{M}$.

Similarly, for any $A_{21} \in \mathscr{M}_{21}$, by using the equation $P_{1}(I+$ $\left.A_{21}\right)=P_{1}$, one can show that

$$
\begin{aligned}
P_{2} A P_{1} & \left(P_{2} L\left(P_{1}\right) P_{2}+P_{1} L\left(P_{1}\right) P_{1}\right) \\
= & \left(P_{2} L\left(P_{1}\right) P_{2}+P_{1} L\left(P_{1}\right) P_{1}\right) P_{2} A P_{1}
\end{aligned}
$$

holds for all $A \in \mathscr{M}$. It follows from (4)-(5) and Lemma 2 that $P_{1} L\left(P_{1}\right) P_{1}+P_{2} L\left(P_{1}\right) P_{2} \in \mathscr{Z}(\mathscr{M})$, as desired.

Let $S=P_{1} L\left(P_{1}\right) P_{2}-P_{2} L\left(P_{1}\right) P_{1}$ and define a map $\delta: \mathscr{M} \rightarrow$ $\mathscr{M}$ by $\delta(A)=L(A)+S A-A S$ for every $A \in \mathscr{M}$. It is easily checked that, for any $A, B \in \mathscr{M}$,

$$
A B=P \Longrightarrow \delta([A, B])=[\delta(A), B]+[A, \delta(B)] .
$$

Moreover, by Claim 1, we have

$$
\begin{aligned}
\delta\left(P_{1}\right) & =L\left(P_{1}\right)-P_{2} L\left(P_{1}\right) P_{1}-P_{1} L\left(P_{1}\right) P_{2} \\
& =P_{1} L\left(P_{1}\right) P_{1}+P_{2} L\left(P_{1}\right) P_{2} \in \mathscr{Z}(\mathscr{M}) .
\end{aligned}
$$

Claim 2. Consider $\delta\left(\mathscr{M}_{i j}\right) \subseteq \mathscr{M}_{i j}, 1 \leq i \neq j \leq 2$. Here we only give the proof for $\mathscr{M}_{12}$. For the case $\mathscr{M}_{21}$, the proof is similar. 
For any $A_{12} \in \mathscr{M}_{12}$, let $\delta\left(A_{12}\right)=S_{11}+S_{12}+S_{21}+S_{22}$. Since $\left(I+A_{12}\right) P_{1}=P_{1}$, we have

$$
\delta\left(\left[I+A_{12}, P_{1}\right]\right)=\left[\delta(I)+\delta\left(A_{12}\right), P_{1}\right]+\left[I+A_{12}, \delta\left(P_{1}\right)\right] .
$$

Note that $\delta(I)=L(I)$. Then, by (1) and (7), the above equation reduces to $S_{11}+2 S_{21}+S_{22}=0$, which implies $S_{11}=S_{21}=S_{22}=$ 0 . Hence $\delta\left(A_{12}\right)=S_{12} \in \mathscr{M}_{12}$.

Claim 3. Consider $\delta(I) \in \mathscr{Z}(\mathscr{M})$. Take any $A_{12} \in \mathscr{M}_{12}$. Since $\left(P_{1}-A_{12}\right)\left(I+A_{12}\right)=P_{1}$, we have

$$
\begin{aligned}
\delta\left(\left[P_{1}-A_{12}, I+A_{12}\right]\right)= & {\left[\delta\left(P_{1}-A_{12}\right), I+A_{12}\right] } \\
& +\left[P_{1}-A_{12}, \delta\left(I+A_{12}\right)\right] .
\end{aligned}
$$

By (7) and Claim 2, the above equation yields $\delta(I) A_{12}=$ $A_{12} \delta(I)$; that is,

$$
\delta(I) P_{1} A P_{2}=P_{1} A P_{2} \delta(I) \quad \forall A \in \mathscr{M} .
$$

Similarly, by the equation $\left(I+A_{21}\right)\left(P_{1}-A_{21}\right)=P_{1}$, one can show that

$$
\delta(I) P_{2} A P_{1}=P_{2} A P_{1} \delta(I) \quad \text { holds } \forall A \in \mathscr{M} .
$$

Equations (10)-(11) and Lemma 2 ensure that $\delta(I) \in \mathscr{Z}(\mathscr{M})$.

Note that, by (7) and Claim 3, one shows

$$
\delta\left(P_{2}\right)=\delta(I)-\delta\left(P_{1}\right) \in \mathscr{Z}(\mathscr{M}) .
$$

Claim 4. For any $A_{i i} \in \mathscr{M}_{i i}$, there exists some map $h_{i}$ : $\mathscr{M}_{i i} \rightarrow \mathscr{Z}(\mathscr{M})$ such that $\delta\left(A_{i i}\right) \in \mathscr{M}_{i i}+h_{i}\left(A_{i i}\right), i=1,2$.

Firstly, take any invertible $A_{11} \in \mathscr{M}_{11}$ and let $\delta\left(A_{11}\right)=$ $S_{11}+S_{12}+S_{21}+S_{22}$. Since $A_{11}^{-1} A_{11}=P_{1}$, we have

$$
0=\delta\left(\left[A_{11}^{-1}, A_{11}\right]\right)=\left[\delta\left(A_{11}^{-1}\right), A_{11}\right]+\left[A_{11}^{-1}, \delta\left(A_{11}\right)\right] .
$$

For any $B_{22} \in \mathscr{M}_{22}$, let $\delta\left(B_{22}\right)=T_{11}+T_{12}+T_{21}+T_{22}$. Since $P_{1}\left(P_{1}+B_{22}\right)=P_{1}$, we have

$$
\begin{aligned}
0 & =\delta\left(\left[P_{1}, P_{1}+B_{22}\right]\right) \\
& =\left[\delta\left(P_{1}\right), P_{1}+B_{22}\right]+\left[P_{1}, \delta\left(P_{1}\right)+\delta\left(B_{22}\right)\right] .
\end{aligned}
$$

By (7), the above equation yields

$$
T_{12}=T_{21}=0 .
$$

Since $\left(A_{11}^{-1}+B_{22}\right) A_{11}=P_{1}$, one gets

$$
\begin{aligned}
0 & =\delta\left(\left[A_{11}^{-1}+B_{22}, A_{11}\right]\right) \\
& =\left[\delta\left(A_{11}^{-1}\right)+\delta\left(B_{22}\right), A_{11}\right]+\left[A_{11}^{-1}+B_{22}, \delta\left(A_{11}\right)\right],
\end{aligned}
$$

which and (13) imply

$$
\begin{array}{r}
\delta\left(B_{22}\right) A_{11}-A_{11} \delta\left(B_{22}\right)+B_{22} \delta\left(A_{11}\right)-\delta\left(A_{11}\right) B_{22}=0 \\
\forall B_{22} \in \mathscr{M}_{22} .
\end{array}
$$

Let $B_{22}=P_{2}$ in (17), and by (12), we get $P_{2} \delta\left(A_{11}\right)=\delta\left(A_{11}\right) P_{2}$, and so

$$
S_{12}=S_{21}=0 \text {. }
$$

Thus, combining (15) and (18), (17) reduces to $T_{11} A_{11}-$ $A_{11} T_{11}+B_{22} S_{22}-S_{22} B_{22}=0$. It follows that

$$
T_{11} A_{11}=A_{11} T_{11}, \quad B_{22} S_{22}=S_{22} B_{22}
$$

holds for all invertible $A_{11} \in \mathscr{M}_{11}$ and all $B_{22} \in \mathscr{M}_{22}$.

Now taking any $A_{11} \in \mathscr{M}_{11}$, there is a scalar $c$ such that $c P_{1}-A_{11}$ is invertible. Then (19) implies $T_{11} A_{11}=A_{11} T_{11}$ for all $A_{11} \in \mathscr{M}_{11}$, and so $T_{11} \in \mathscr{Z}\left(\mathscr{M}_{11}\right)$. Note that, for $T_{11} \in \mathscr{Z}\left(\mathscr{M}_{11}\right)$, there must be some $h_{2}\left(B_{22}\right) \in \mathscr{Z}(\mathscr{M})$ such that $h_{2}\left(B_{22}\right)-T_{11} \in \mathscr{Z}\left(\mathscr{M}_{22}\right)$. So

$$
\begin{aligned}
\delta\left(B_{22}\right)= & T_{11}+T_{22} \\
= & T_{22}+\left(T_{11}-h_{2}\left(B_{22}\right)\right) \\
& +h_{2}\left(B_{22}\right) \in \mathscr{M}_{22}+h_{2}\left(B_{22}\right) .
\end{aligned}
$$

On the other hand, by (19) and the arbitrariness of $B_{22} \epsilon$ $\mathscr{M}_{22}$, one can show that $S_{22} \in \mathscr{Z}\left(\mathscr{M}_{22}\right)$, and so $\delta\left(A_{11}\right)=$ $S_{11}+S_{22} \in \mathscr{M}_{11}+\mathscr{Z}\left(\mathscr{M}_{22}\right)$ for all invertible $A_{11} \in \mathscr{M}_{11}$. Now consider any $A_{11} \in \mathscr{M}_{11}$. There is a scalar $c$ such that $c P_{1}-A_{11}$ is invertible. Also note that $c P_{1}$ is invertible in $\mathscr{M}_{11}$. Hence $\delta\left(A_{11}\right)=P_{1} \delta\left(A_{11}\right) P_{1}+P_{2} \delta\left(A_{11}\right) P_{2} \in \mathscr{M}_{11}+\mathscr{Z}\left(\mathscr{M}_{22}\right)$ for all $A_{11} \in \mathscr{M}_{11}$. Again, for $P_{2} \delta\left(A_{11}\right) P_{2} \in \mathscr{Z}\left(\mathscr{M}_{22}\right)$, there is some $h_{1}\left(A_{11}\right) \in \mathscr{Z}(\mathscr{M})$ such that $h_{1}\left(A_{11}\right)-P_{2} \delta\left(A_{11}\right) P_{2} \in \mathscr{Z}\left(\mathscr{M}_{11}\right)$. It follows that

$$
\begin{aligned}
\delta\left(A_{11}\right)= & P_{1} \delta\left(A_{11}\right) P_{1}+P_{2} \delta\left(A_{11}\right) P_{2} \\
= & P_{1} \delta\left(A_{11}\right) P_{1}+\left(P_{2} \delta\left(A_{11}\right) P_{2}-h_{1}\left(A_{11}\right)\right) \\
& +h_{1}\left(A_{11}\right) \in \mathscr{M}_{11}+h_{1}\left(A_{11}\right) .
\end{aligned}
$$

Claim 4 is true.

Now define two maps $h: \mathscr{M} \rightarrow \mathscr{E}(\mathscr{M})$ and $d: \mathscr{M} \rightarrow$ $\mathscr{M}$, respectively, by

$$
h(A)=h_{1}\left(A_{11}\right)+h_{2}\left(A_{22}\right), \quad d(A)=\delta(A)-h(A)
$$

for all $A=A_{11}+A_{12}+A_{21}+A_{22} \in \mathscr{M}$. Then by Claims 2 and 4 , we have

$$
\begin{aligned}
d\left(\mathscr{M}_{i j}\right) & \subseteq \mathscr{M}_{i j}, \\
d\left(\mathscr{M}_{i i}\right) & \subseteq \mathscr{M}_{i i}, \\
d\left(\mathscr{M}_{i j}\right) & =\delta\left(\mathscr{M}_{i j}\right), \\
1 & \leq i \neq j \leq 2 .
\end{aligned}
$$


Claim 5. $d$ and $h$ are additive. By the definitions of $d$ and $h$, we only need to verify that $d$ is additive on $\mathscr{M}_{i i}(i=1,2)$. In fact, for any $A_{11}, B_{11} \in \mathscr{M}_{11}$, we have

$$
\begin{aligned}
d\left(A_{11}\right. & \left.+B_{11}\right)+h\left(A_{11}+B_{11}\right) \\
= & \delta\left(A_{11}+B_{11}\right)=\delta\left(A_{11}\right)+\delta\left(B_{11}\right) \\
= & d\left(A_{11}\right)+h\left(A_{11}\right)+d\left(B_{11}\right)+h\left(B_{11}\right) \\
= & d\left(A_{11}\right)+d\left(B_{11}\right)+\left(h\left(A_{11}\right)+h\left(B_{11}\right)\right) .
\end{aligned}
$$

That is,

$$
\begin{aligned}
& d\left(A_{11}+B_{11}\right)-\left(d\left(A_{11}\right)+d\left(B_{11}\right)\right) \\
& =h\left(A_{11}\right)+h\left(B_{11}\right)-h\left(A_{11}+B_{11}\right) \in \mathscr{Z}(\mathscr{M}) .
\end{aligned}
$$

Since $\mathscr{M}_{11} \cap \mathscr{Z}(\mathscr{M})=\{0\}$ by Lemma 3 , one sees $d\left(A_{11}+B_{11}\right)=$ $d\left(A_{11}\right)+d\left(B_{11}\right)$, and consequently, $h\left(A_{11}\right)+h\left(B_{11}\right)=h\left(A_{11}+\right.$ $\left.B_{11}\right)$.

Similarly, one can prove that $d$ is additive on $\mathscr{M}_{22}$.

Claim 6. $d$ is a derivation; that is, $d(A B)=d(A) B+A d(B)$ for all $A, B \in \mathscr{M}$.

We will complete the proof of the claim by three steps.

Step 1. For any $A_{i i} \in \mathscr{M}_{i i}, A_{i j}, B_{i j} \in \mathscr{M}_{i j}$, and $B_{j j} \in \mathscr{M}_{j j}$, we have $d\left(A_{i i} B_{i j}\right)=d\left(A_{i i}\right) B_{i j}+A_{i i} d\left(B_{i j}\right)$ and $d\left(A_{i j} B_{j j}\right)=$ $d\left(A_{i j}\right) B_{j j}+A_{i j} d\left(B_{j j}\right), 1 \leq i \neq j \leq 2$.

In fact, for any invertible $A_{11} \in \mathscr{M}_{11}$ and any $B_{12} \in \mathscr{M}_{12}$, since $\left(A_{11}^{-1}+B_{12}\right) A_{11}=P_{1}$, by (23) and (13), we have

$$
\begin{aligned}
-d\left(A_{11} B_{12}\right)= & -\delta\left(A_{11} B_{12}\right)=\delta\left(\left[A_{11}^{-1}+B_{12}, A_{11}\right]\right) \\
= & {\left[\delta\left(A_{11}^{-1}\right)+\delta\left(B_{12}\right), A_{11}\right] } \\
& +\left[A_{11}^{-1}+B_{12}, \delta\left(A_{11}\right)\right] \\
= & {\left[\delta\left(A_{11}^{-1}\right), A_{11}\right]+\left[\delta\left(B_{12}\right), A_{11}\right] } \\
& +\left[A_{11}^{-1}, \delta\left(A_{11}\right)\right]+\left[B_{12}, \delta\left(A_{11}\right)\right] \\
= & {\left[d\left(B_{12}\right), A_{11}\right]+\left[B_{12}, d\left(A_{11}\right)\right] } \\
= & -A_{11} d\left(B_{12}\right)-d\left(A_{11}\right) B_{12} .
\end{aligned}
$$

That is,

$$
d\left(A_{11} B_{12}\right)=d\left(A_{11}\right) B_{12}+A_{11} d\left(B_{12}\right)
$$

for any invertible $A_{11} \in \mathscr{M}_{11}$ and all $B_{12} \in \mathscr{M}_{12}$.

Take any $A_{11} \in \mathscr{M}_{11}$. There is a scalar $c$ such that $c P_{1}-A_{11}$ is invertible. Note that $c P_{1}$ is also invertible in $\mathscr{M}_{11}$. So by (27) one gets

$$
\begin{gathered}
d\left(c P_{1} B_{12}\right)=d\left(c P_{1}\right) B_{12}+c P_{1} d\left(B_{12}\right) \\
d\left(\left(c P_{1}-A_{11}\right) B_{12}\right) \\
=d\left(c P_{1}-A_{11}\right) B_{12}+\left(c P_{1}-A_{11}\right) d\left(B_{12}\right) .
\end{gathered}
$$

Hence, $d\left(A_{11} B_{12}\right)=d\left(A_{11}\right) B_{12}+A_{11} d\left(B_{12}\right)$ holds for all $A_{11} \in \mathscr{M}_{11}$ and all $B_{12} \in \mathscr{M}_{12}$.

Similarly, by $B_{11}\left(A_{21}+B_{11}^{-1}\right)=P_{1}$, one can show that $d\left(A_{21} B_{11}\right)=d\left(A_{21}\right) B_{11}+A_{21} d\left(B_{11}\right)$ holds for all $A_{21} \in \mathscr{M}_{21}$ and $B_{11} \in \mathscr{M}_{11}$; by $\left(P_{1}+A_{22}-A_{22} B_{21}\right)\left(P_{1}+B_{21}\right)=P_{1}$, one can show that $d\left(A_{22} B_{21}\right)=d\left(A_{22}\right) B_{21}+A_{22} d\left(B_{21}\right)$ holds for all $A_{22} \in \mathscr{M}_{22}$ and $B_{21} \in \mathscr{M}_{21}$; and by using the relation $\left(P_{1}+A_{12}\right)\left(P_{1}+B_{22}-A_{12} B_{22}\right)=P_{1}$, one can obtain that $d\left(A_{12} B_{22}\right)=d\left(A_{12}\right) B_{22}+A_{12} d\left(B_{22}\right)$ holds for all $A_{12} \in \mathscr{M}_{12}$ and $B_{22} \in \mathscr{M}_{22}$.

Step 2. For any $A_{i i}, B_{i i} \in \mathscr{M}_{i i}$, we have $d\left(A_{i i} B_{i i}\right)=d\left(A_{i i}\right) B_{i i}+$ $A_{i i} d\left(B_{i i}\right), i=1,2$.

Let $i \neq j$. For any $A_{i i}, B_{i i} \in \mathscr{M}_{i i}$ and any $S_{i j} \in \mathscr{M}_{i j}$, by Step 1, on the one hand, we have

$$
d\left(A_{i i} B_{i i} S_{i j}\right)=d\left(A_{i i} B_{i i}\right) S_{i j}+A_{i i} B_{i i} d\left(S_{i j}\right)
$$

on the other hand,

$$
\begin{aligned}
d\left(A_{i i} B_{i i} S_{i j}\right) & =d\left(A_{i i}\right) B_{i i} S_{i j}+A_{i i} d\left(B_{i i} S_{i j}\right) \\
& =d\left(A_{i i}\right) B_{i i} S_{i j}+A_{i i} d\left(B_{i j}\right) S_{i j}+A_{i i} B_{i i} d\left(S_{i j}\right) .
\end{aligned}
$$

Comparing the above two equations gets that $\left(d\left(A_{i i} B_{i i}\right)-\right.$ $\left.d\left(A_{i i}\right) B_{i i}-A_{i i} d\left(B_{i i}\right)\right) S_{i j}=0$ holds for all $S_{i j} \in \mathscr{M}_{i j}$; that is,

$$
\left(d\left(A_{i i} B_{i i}\right)-d\left(A_{i i}\right) B_{i i}-A_{i i} d\left(B_{i i}\right)\right) P_{i} S P_{j}=0
$$

holds for all $S \in \mathscr{M}$. Note that $\overline{P_{j}}=I$. It follows from the definition of the central carrier that $\operatorname{span}\left\{T P_{j}(x): T \in\right.$ $\mathscr{M}, x \in H\}$ is dense in $H$. It follows from (23) that $d\left(A_{i i} B_{i i}\right)=$ $d\left(A_{i i}\right) B_{i i}+A_{i i} d\left(B_{i i}\right)$ holds for all $A_{i i}, B_{i i} \in \mathscr{M}_{i i}$, as desired.

Note that, by using Step 2 and the fact that $d\left(\mathscr{M}_{i i}\right) \subseteq \mathscr{M}_{i i}$ $(i=1,2)$, one can get

$$
d\left(P_{1}\right)=d\left(P_{2}\right)=0 \text {. }
$$

Step 3. Consider $d\left(A_{i j} B_{j i}\right)=d\left(A_{i j}\right) B_{j i}+A_{i j} d\left(B_{j i}\right)$ for all $A_{i j} \epsilon$ $\mathscr{M}_{i j}$ and $B_{j i} \in \mathscr{M}_{j i}, 1 \leq i \neq j \leq 2$.

Take any $A_{12} \in \mathscr{M}_{12}$ and $B_{21} \in \mathscr{M}_{21}$. Since $\left(P_{1}+A_{12}-\right.$ $\left.A_{12} B_{21}\right)\left(P_{1}+B_{21}\right)=P_{1}$, by the definition of $d$, Claim 5, (23), and (32), we have

$$
\begin{aligned}
d\left(A_{12} B_{21}-A_{12}-B_{21}-B_{21} A_{12}+B_{21} A_{12} B_{21}\right) \\
\quad+h\left(A_{12} B_{21}-B_{21} A_{12}\right) \\
=\delta\left(A_{12} B_{21}-A_{12}-B_{21}-B_{21} A_{12}+B_{21} A_{12} B_{21}\right) \\
=\delta\left(\left[P_{1}+A_{12}-A_{12} B_{21}, P_{1}+B_{21}\right]\right)
\end{aligned}
$$




$$
\begin{aligned}
= & {\left[\delta\left(P_{1}+A_{12}-A_{12} B_{21}\right), P+B_{21}\right] } \\
& +\left[P_{1}+A_{12}-A_{12} B_{21}, \delta\left(P_{1}+B_{21}\right)\right] \\
= & {\left[d\left(P_{1}+A_{12}-A_{12} B_{21}\right), P_{1}+B_{21}\right] } \\
& +\left[P_{1}+A_{12}-A_{12} B_{21}, d\left(P_{1}+B_{21}\right)\right] \\
= & d\left(A_{12}\right) B_{21}-d\left(A_{12}\right)-B_{21} d\left(A_{12}\right)+B_{21} d\left(A_{12} B_{21}\right) \\
& +A_{12} d\left(B_{21}\right)-d\left(B_{21}\right)+d\left(B_{21}\right) A_{12} B_{21}-d\left(B_{21}\right) A_{12} .
\end{aligned}
$$

It follows from Step 1 that

$$
\begin{aligned}
& d\left(A_{12} B_{21}-B_{21} A_{12}\right)+h\left(A_{12} B_{21}-B_{21} A_{12}\right) \\
& \quad=d\left(A_{12}\right) B_{21}-B_{21} d\left(A_{12}\right)+A_{12} d\left(B_{21}\right)-d\left(B_{21}\right) A_{12} .
\end{aligned}
$$

Multiplying by $A_{12}$ from the left side and the right side, respectively, in (34) and applying (23), we get

$$
\begin{gathered}
A_{12} d\left(B_{21} A_{12}\right)-h\left(A_{12} B_{21}-B_{21} A_{12}\right) A_{12} \\
=A_{12} B_{21} d\left(A_{12}\right)+A_{12} d\left(B_{21}\right) A_{12}, \\
d\left(A_{12} B_{21}\right) A_{12}+h\left(A_{12} B_{21}-B_{21} A_{12}\right) A_{12} \\
=d\left(A_{12}\right) B_{21} A_{12}+A_{12} d\left(B_{21}\right) A_{12} .
\end{gathered}
$$

These two equations, together with Step 1, yield

$$
\begin{gathered}
-d\left(A_{12}\right) B_{21} A_{12}-h\left(A_{12} B_{21}-B_{21} A_{12}\right) A_{12} \\
=-d\left(A_{12} B_{21}\right) A_{12}+A_{12} d\left(B_{21}\right) A_{12}, \\
-A_{12} B_{21} d\left(A_{12}\right)+h\left(A_{12} B_{21}-B_{21} A_{12}\right) A_{12} \\
=-A_{12} d\left(B_{21} A_{12}\right)+A_{12} d\left(B_{21}\right) A_{12} .
\end{gathered}
$$

Comparing the above two equations, one achieves

$$
h\left(A_{12} B_{21}-B_{21} A_{12}\right) A_{12}=0 \text {. }
$$

Similarly, multiplying by $B_{21}$ from the left side and the right side, respectively, in (34), one can verify

$$
h\left(A_{12} B_{21}-B_{21} A_{12}\right) B_{21}=0 .
$$

Next, we will prove $h\left(A_{12} B_{21}-B_{21} A_{12}\right)=0$. To do this, for any $A_{12}$, let $A_{12}=V\left|A_{12}\right|$ be its polar decomposition. Then (37) implies $h\left(A_{12} B_{21}-B_{21} A_{12}\right)\left|A_{12}\right|=0$, and so $\left|A_{12}\right| h\left(A_{12} B_{21}-B_{21} A_{12}\right)^{*}=0$. It follows that

$$
\begin{aligned}
A_{12} h & \left(A_{12} B_{21}-B_{21} A_{12}\right)^{*} \\
& =V\left|A_{12}\right| h\left(A_{12} B_{21}-B_{21} A_{12}\right)^{*}=0 .
\end{aligned}
$$

Similarly, one can show

$$
B_{21} h\left(A_{12} B_{21}-B_{21} A_{12}\right)^{*}=0 .
$$

Multiplying by $h\left(A_{12} B_{21}-B_{21} A_{12}\right)^{*}$ from the right side in (34), by using (39)-(40), we get

$$
\begin{aligned}
& d\left(A_{12} B_{21}-B_{21} A_{12}\right) h\left(A_{12} B_{21}-B_{21} A_{12}\right)^{*} \\
& \quad+h\left(A_{12} B_{21}-B_{21} A_{12}\right) h\left(A_{12} B_{21}-B_{21} A_{12}\right)^{*}=0 .
\end{aligned}
$$

Note that, by Step 2, (23), and (39)-(40), we have

$$
\begin{array}{rl}
d\left(A_{12} B_{21}\right) h\left(A_{12} B_{21}-B_{21} A_{12}\right)^{*} & d\left(A_{12} B_{21} P_{1} h\left(A_{12} B_{21}-B_{21} A_{12}\right)^{*} P_{1}\right) \\
= & -A_{12} B_{21} d\left(P_{1} h\left(A_{12} B_{21}-B_{21} A_{12}\right)^{*} P_{1}\right) \\
= & -A_{12} B_{21} d\left(P_{1} h\left(A_{12} B_{21}-B_{21} A_{12}\right)^{*} P_{1}\right), \\
d\left(B_{21} A_{12}\right) h\left(A_{12} B_{21}-B_{21} A_{12}\right)^{*} \\
=d\left(B_{21} A_{12} P_{2} h\left(A_{12} B_{21}-B_{21} A_{12}\right)^{*} P_{2}\right) \\
\quad-B_{21} A_{12} d\left(P_{2} h\left(A_{12} B_{21}-B_{21} A_{12}\right)^{*} P_{2}\right) \\
=-B_{21} A_{12} d\left(P_{2} h\left(A_{12} B_{21}-B_{21} A_{12}\right)^{*} P_{2}\right) .
\end{array}
$$

Hence, (41) implies

$$
\begin{aligned}
& h\left(A_{12} B_{21}-B_{21} A_{12}\right)^{*} \\
& \quad \times h\left(A_{12} B_{21}-B_{21} A_{12}\right) h\left(A_{12} B_{21}-B_{21} A_{12}\right)^{*}=0,
\end{aligned}
$$

and so $h\left(A_{12} B_{21}-B_{21} A_{12}\right)=0$. Thus, (34) reduces to

$$
\begin{aligned}
& d\left(A_{12} B_{21}-B_{21} A_{12}\right) \\
& \quad=d\left(A_{12}\right) B_{21}-B_{21} d\left(A_{12}\right)+A_{12} d\left(B_{21}\right)-d\left(B_{21}\right) A_{12},
\end{aligned}
$$

which implies that $d\left(A_{12} B_{21}\right)=d\left(A_{12}\right) B_{21}+A_{12} d\left(B_{21}\right)$ and $d\left(B_{21} A_{12}\right)=B_{21} d\left(A_{12}\right)+d\left(B_{21}\right) A_{12}$ hold for all $A_{12} \in \mathscr{M}_{12}$ and $B_{21} \in \mathscr{M}_{21}$, as desired.

Now combining Steps $1-3$, it is easily checked that $d$ is an additive derivation.

Claim 7. Consider $h([A, B])=0$ for all $A, B \in \mathscr{M}$ with $A B=$ $P_{1}$.

In fact, for any $A, B \in \mathscr{M}$ with $A B=P_{1}$, we have

$$
\begin{aligned}
h([A, B])= & \delta([A, B])-d([A, B]) \\
= & {[\delta(A), B]+[A, \delta(B)]-d(A B-B A) } \\
= & {[d(A)-h(A), B] } \\
& +[A, d(B)-h(B)]-d(A B-B A) \\
= & {[d(A), B]+[A, d(B)]-d(A B-B A)=0 . }
\end{aligned}
$$

Claim 8. The theorem holds. 
Indeed, let $\varphi(A)=d(A)-(S A-A S)$ for all $A \in \mathscr{M}$; then, by the definitions of $\delta$ and $d$, we have $L(A)=\varphi(A)+h(A)$ for all $A \in \mathscr{M}$. It is easy to check that $\varphi$ is an additive derivation on $\mathscr{M}$.

The proof is finished.

\section{Characterizing $\xi$-Lie Derivations by Local Action}

In this section, we will give a complete characterization of additive $\xi$-Lie derivations for $\xi \neq 1$ by local action at some core-free projection $P$ with $\bar{P}=I$ on general von Neumann algebras having no central summands of type $I_{1}$.

The following is the main result of this section.

Theorem 4. Let $\mathscr{M}$ be a von Neumann algebra without central summands of type $I_{1}$ and $P \in M$ a nonzero core-free projection with $\bar{P}=I$. Suppose that $L: \mathscr{M} \rightarrow \mathscr{M}$ is an additive map and $\xi$ is a scalar with $\xi \neq 1$. Then $L$ satisfies $L\left([A, B]_{\xi}\right)=$ $[L(A), B]_{\xi}+[A, L(B)]_{\xi}$ for any $A, B \in \mathscr{M}$ with $A B=P$ if and only if $L$ is an additive derivation and $L(\xi A)=\xi L(A)$ for all $A \in \mathscr{M}$.

Proof. Still, the "if" part is clear. For the "only if" part, we use the same symbols as that in the proof of Theorem 1 . In the sequel, we always assume that $\xi \neq 1$ and $L: \mathscr{M} \rightarrow \mathscr{M}$ is an additive map satisfying $L\left([A, B]_{\xi}\right)=[L(A), B]_{\xi}+[A, L(B)]_{\xi}$ for $A, B \in \mathscr{M}$ with $A B=P=P_{1}$. We will prove the "only if" part by several claims.

Claim 1. Consider $P_{1} L(I) P_{2}=P_{2} L(I) P_{1}=0$ and $P_{2} L\left(P_{1}\right) P_{2}=$ $P_{1} L\left(P_{2}\right) P_{1}=0$. Since $P_{1} P_{1}=P_{1}$, we have $L\left(\left[P_{1}, P_{1}\right]_{\xi}\right)=$ $\left[L\left(P_{1}\right), P_{1}\right]_{\xi}+\left[P_{1}, L\left(P_{1}\right)\right]_{\xi}$; that is,

$$
\begin{aligned}
& L\left(P_{1}\right)-L\left(\xi P_{1}\right) \\
& \quad=L\left(P_{1}\right) P_{1}-\xi P_{1} L\left(P_{1}\right)+P_{1} L\left(P_{1}\right)-\xi L\left(P_{1}\right) P_{1} .
\end{aligned}
$$

Multiplying by $P_{i}$ and $P_{j}(1 \leq i \neq j \leq 2)$ from the left and the right sides, respectively, in (47), one gets

$$
P_{i} L\left(\xi P_{1}\right) P_{j}=\xi P_{i} L\left(P_{1}\right) P_{j}, \quad 1 \leq i \neq j \leq 2 .
$$

As $P_{1} I=I P_{1}=P_{1}, L\left(\left[P_{1}, I\right]_{\xi}\right)=\left[L\left(P_{1}\right), I\right]_{\xi}+\left[P_{1}, L(I)\right]_{\xi}$, and $L\left(\left[I, P_{1}\right]_{\xi}\right)=\left[L(I), P_{1}\right]_{\xi}+\left[I, L\left(P_{1}\right)\right]_{\xi}$, that is,

$$
\begin{aligned}
& L\left(P_{1}\right)-L\left(\xi P_{1}\right)=L\left(P_{1}\right)-\xi L\left(P_{1}\right)+P_{1} L(I)-\xi L(I) P_{1}, \\
& L\left(P_{1}\right)-L\left(\xi P_{1}\right)=L(I) P_{1}-\xi P_{1} L(I)+L\left(P_{1}\right)-\xi L\left(P_{1}\right) .
\end{aligned}
$$

Multiplying by $P_{1}$ and $P_{2}$ from the left and the right sides, respectively, in (49) and combining with (48), one gets $P_{1} L(I) P_{2}=0$; multiplying by $P_{2}$ and $P_{1}$ from the left and the right sides, respectively, in (50) and combining with (48), one gets $P_{2} L(I) P_{1}=0$.

On the other hand, since $\left(P_{1}-P_{2}\right) P_{1}=P_{1}$, we have $L\left(\left[P_{1}-\right.\right.$ $\left.\left.P_{2}, P_{1}\right]_{\xi}\right)=\left[L\left(P_{1}\right)-L\left(P_{2}\right), P_{1}\right]_{\xi}+\left[P_{1}-P_{2}, L\left(P_{1}\right)\right]_{\xi}$, which and (47) yield

$$
L\left(P_{2}\right) P_{1}-\xi P_{1} L\left(P_{2}\right)+P_{2} L\left(P_{1}\right)-\xi L\left(P_{1}\right) P_{2}=0 .
$$

This implies $(1-\xi) P_{1} L\left(P_{2}\right) P_{1}=0$ and $(1-\xi) P_{2} L\left(P_{1}\right) P_{2}=$ 0 . It follows from the fact that $\xi \neq 1$ that $P_{1} L\left(P_{2}\right) P_{1}=0$ and $P_{2} L\left(P_{1}\right) P_{2}=0$. Claim 1 is true.

Define a map $\delta: \mathscr{M} \rightarrow \mathscr{M}$ by $\delta(A)=L(A)+S A-A S$ for each $A \in \mathscr{M}$, where $S=P_{1} L\left(P_{1}\right) P_{2}-P_{2} L\left(P_{1}\right) P_{1}$. It is easily verified that $\delta$ is also an additive map satisfying

$$
\delta\left([A, B]_{\xi}\right)=[\delta(A), B]_{\xi}+[A, \delta(B)]_{\xi}
$$$$
\text { for any } A, B \in \mathscr{M} \text { with } A B=P=P_{1} \text {. }
$$

Moreover, $P_{1} \delta(I) P_{2}=P_{2} \delta(I) P_{1}=P_{2} \delta\left(P_{1}\right) P_{2}=P_{1} \delta\left(P_{2}\right) P_{1}=0$ by Claim 1 . Thus we obtain

$$
\begin{aligned}
\delta\left(P_{1}\right) & =L\left(P_{1}\right)+S P_{1}-P_{1} S=P_{1} L\left(P_{1}\right) P_{1} \\
& =P_{1} \delta\left(P_{1}\right) P_{1}-P_{1}\left(S P_{1}-P_{1} S\right) P_{1}=P_{1} \delta\left(P_{1}\right) P_{1} \in \mathscr{M}_{11}, \\
\delta\left(P_{2}\right) & =L\left(P_{2}\right)+S P_{2}-P_{2} S=P_{2} L\left(P_{2}\right) P_{2} \\
& =P_{2} \delta\left(P_{2}\right) P_{2}-P_{2}\left(S P_{2}-P_{2} S\right) P_{2}=P_{2} \delta\left(P_{2}\right) P_{2} \in \mathscr{M}_{22} .
\end{aligned}
$$

Note that (47) is also true for the map $\delta$. So, by (53), one can also get

$$
\delta\left(P_{1}\right)+\delta\left(\xi P_{1}\right)-2 \xi \delta\left(P_{1}\right)=0 .
$$

Claim 2. Consider $\delta\left(\mathscr{M}_{i i}\right) \subseteq \mathscr{M}_{i i}, i=1,2$. For any $A_{22} \in$ $\mathscr{M}_{22}$, since $P_{1}\left(P_{1}+A_{22}\right)=P_{1}$, we have

$$
\begin{aligned}
& \delta\left(\left[P_{1}, P_{1}+A_{22}\right]_{\xi}\right) \\
& \quad=\left[\delta\left(P_{1}\right), P_{1}+A_{22}\right]_{\xi}+\left[P_{1}, \delta\left(P_{1}\right)+\delta\left(A_{22}\right)\right]_{\xi} .
\end{aligned}
$$

By (53) and (55), the above equation reduces to

$$
P_{1} \delta\left(A_{22}\right)-\xi \delta\left(A_{22}\right) P_{1}=0 .
$$

Multiplying by $P_{2}$ from the right side in (57), one gets $P_{1} \delta\left(A_{22}\right) P_{2}=0$; multiplying by $P_{1}$ from both sides in (57), one gets $P_{1} \delta\left(A_{22}\right) P_{1}=0$.

Similarly, by $\left(P_{1}+A_{22}\right) P_{1}=P_{1}$, one can show $P_{2} \delta\left(A_{22}\right) P_{1}=0$. Thus, we have proved that $\delta\left(A_{22}\right) \in \mathscr{M}_{22}$ for all $A_{22} \in \mathscr{M}_{22}$.

Next, take any invertible $A_{11} \in \mathscr{M}_{11}$. By $A_{11} A_{11}^{-1}=P_{1}$ and $A_{11}\left(A_{11}^{-1}+P_{2}\right)=P_{1}$, we get

$$
\begin{aligned}
\delta\left(P_{1}\right)-\delta\left(\xi P_{1}\right) & =\delta\left(\left[A_{11}, A_{11}^{-1}\right]_{\xi}\right) \\
= & {\left[\delta\left(A_{11}\right), A_{11}^{-1}\right]_{\xi}+\left[A_{11}, \delta\left(A_{11}^{-1}\right)\right]_{\xi}, } \\
\delta\left(P_{1}\right)-\delta\left(\xi P_{1}\right)= & \delta\left(\left[A_{11}, A_{11}^{-1}+P_{2}\right]_{\xi}\right) \\
= & {\left[\delta\left(A_{11}\right), A_{11}^{-1}+P_{2}\right]_{\xi} } \\
& +\left[A_{11}, \delta\left(A_{11}^{-1}\right)+\delta\left(P_{2}\right)\right]_{\xi}
\end{aligned}
$$


Comparing the two above equations, and by (54), one has $\delta\left(A_{11}\right) P_{2}-\xi P_{2} \delta\left(A_{11}\right)=0$, which implies $P_{1} \delta\left(A_{11}\right) P_{2}=P_{2} \delta$ $\left(A_{11}\right) P_{2}=0$ as $\xi \neq 1$.

Similarly, by $A_{11}^{-1} A_{11}=P_{1}$ and $\left(A_{11}^{-1}+P_{2}\right) A_{11}=P_{1}$, one can prove $P_{2} \delta\left(A_{11}\right) P_{1}=0$.

Thus, we have proved that $\delta\left(A_{11}\right) \in \mathscr{M}_{11}$ if $A_{11} \in \mathscr{M}_{11}$ is invertible. Now for any $A_{11} \in \mathscr{M}_{11}$, we can find a scalar $c$ such that $c P_{1}-A_{11}$ is invertible in $\mathscr{M}_{11}$. It follows from the preceding case that $\delta\left(c P_{1}-A_{11}\right), \delta\left(c P_{1}\right) \in \mathscr{M}_{11}$. Therefore, $\delta\left(A_{11}\right)=\delta\left(c P_{1}\right)-\delta\left(c P_{1}-A_{11}\right) \in \mathscr{M}_{11}$ for each $A_{11} \in \mathscr{M}_{11}$. Claim 2 holds.

Claim 3. For any $A_{i j} \in \mathscr{M}_{i j}, 1 \leq i \neq j \leq 2$, we have $\delta\left(P_{i}\right) A_{i j}=$ $A_{i j} \delta\left(P_{j}\right)$. Moreover, the following statements hold.

(1) If $\xi \neq-1$, then $\delta\left(A_{i j}\right) \in \mathscr{M}_{i j}$.

(2) If $\xi=-1$, then $P_{1} \delta\left(A_{i j}\right) P_{1}=P_{2} \delta\left(A_{i j}\right) P_{2}=0$.

First, consider the case $A_{12} \in \mathscr{M}_{12}$. Since $\left(P_{1}+A_{12}\right) P_{1}=$ $P_{1}$, we have

$$
\begin{aligned}
& \delta\left(\left[P_{1}+A_{12}, P_{1}\right]_{\xi}\right) \\
& \quad=\left[\delta\left(P_{1}\right)+\delta\left(A_{12}\right), P_{1}\right]_{\xi}+\left[P_{1}+A_{12}, \delta\left(P_{1}\right)\right]_{\xi}
\end{aligned}
$$

By (53) and (55), we get

$$
-\delta\left(\xi A_{12}\right)=\delta\left(A_{12}\right) P_{1}-\xi P_{1} \delta\left(A_{12}\right)-\xi \delta\left(P_{1}\right) A_{12} .
$$

Multiplying by $P_{2}$ and $P_{1}$ from the left and the right sides, respectively, in (60), we get $P_{2} \delta\left((1+\xi) A_{12}\right) P_{1}=0$. Since $A_{12}$ is arbitrary, replacing $A_{12}$ by $(1+\xi)^{-1} A_{12}$ if $\xi \neq-1$, one achieves

$$
P_{2} \delta\left(A_{12}\right) P_{1}=0 \quad \text { for the case } \xi \neq-1 .
$$

Since $\left(P_{1}+A_{12}\right)\left(I-A_{12}\right)=P_{1}$, we have

$$
\begin{aligned}
\delta\left(\left[P_{1}+A_{12}, I-A_{12}\right]_{\xi}\right)= & {\left[\delta\left(P_{1}\right)+\delta\left(A_{12}\right), I-A_{12}\right]_{\xi} } \\
& +\left[P_{1}+A_{12}, \delta(I)-\delta\left(A_{12}\right)\right]_{\xi} .
\end{aligned}
$$

That is,

$$
\begin{aligned}
\delta\left(P_{1}\right) & -\delta\left(\xi P_{1}\right)-\delta\left(\xi A_{12}\right) \\
= & \delta\left(P_{1}\right)-\delta\left(P_{1}\right) A_{12}+\delta\left(A_{12}\right)-\delta\left(A_{12}\right) A_{12} \\
& -\xi\left(\delta\left(P_{1}\right)+\delta\left(A_{12}\right)-A_{12} \delta\left(P_{1}\right)-A_{12} \delta\left(A_{12}\right)\right) \\
& +P_{1} \delta(I)-P_{1} \delta\left(A_{12}\right)+A_{12} \delta(I)-A_{12} \delta\left(A_{12}\right) \\
& -\xi\left(\delta(I) P_{1}+\delta(I) A_{12}-\delta\left(A_{12}\right) P_{1}-\delta\left(A_{12}\right) A_{12}\right) .
\end{aligned}
$$

Since $\left(P_{1}-A_{12}\right)\left(I+A_{12}\right)=P_{1}$, we have

$$
\begin{aligned}
\delta\left(\left[P_{1}-A_{12}, I+A_{12}\right]_{\xi}\right)= & {\left[\delta\left(P_{1}\right)-\delta\left(A_{12}\right), I+A_{12}\right]_{\xi} } \\
& +\left[P_{1}-A_{12}, \delta(I)+\delta\left(A_{12}\right)\right]_{\xi} .
\end{aligned}
$$

That is,

$$
\begin{aligned}
\delta\left(P_{1}\right) & -\delta\left(\xi P_{1}\right)+\delta\left(\xi A_{12}\right) \\
= & \delta\left(P_{1}\right)+\delta\left(P_{1}\right) A_{12}-\delta\left(A_{12}\right)-\delta\left(A_{12}\right) A_{12} \\
& -\xi\left(\delta\left(P_{1}\right)-\delta\left(A_{12}\right)+A_{12} \delta\left(P_{1}\right)-A_{12} \delta\left(A_{12}\right)\right) \\
& +P_{1} \delta(I)+P_{1} \delta\left(A_{12}\right)-A_{12} \delta(I)-A_{12} \delta\left(A_{12}\right) \\
& -\xi\left(\delta(I) P_{1}-\delta(I) A_{12}+\delta\left(A_{12}\right) P_{1}-\delta\left(A_{12}\right) A_{12}\right) .
\end{aligned}
$$

Comparing (63)-(65) and by (53)-(54), one can obtain

$$
\begin{aligned}
-\delta\left(\xi A_{12}\right)= & -\delta\left(P_{1}\right) A_{12}+\delta\left(A_{12}\right)-\xi \delta\left(A_{12}\right) \\
& -P_{1} \delta\left(A_{12}\right)+A_{12} \delta\left(P_{2}\right) \\
& -\xi \delta\left(P_{1}\right) A_{12}+\xi \delta\left(A_{12}\right) P_{1} .
\end{aligned}
$$

Then, (60) and (66) yield

$$
\begin{aligned}
& (1-\xi) \delta\left(A_{12}\right) P_{1}+(1-\xi) P_{1} \delta\left(A_{12}\right) \\
& \quad-(1-\xi) \delta\left(A_{12}\right)+\delta\left(P_{1}\right) A_{12}-A_{12} \delta\left(P_{2}\right)=0 .
\end{aligned}
$$

Multiplying by $P_{i}(i=1,2)$ from both sides in (67), and by (53)-(54), we have

$$
P_{1} \delta\left(A_{12}\right) P_{1}=0, \quad P_{2} \delta\left(A_{12}\right) P_{2}=0 ;
$$

multiplying by $P_{1}$ and $P_{2}$ and from the left and the right sides, respectively, in (67), we have

$$
\delta\left(P_{1}\right) A_{12}=A_{12} \delta\left(P_{2}\right) .
$$

Now combining (61) and (68)-(69), we achieve that the claim holds for any $A_{12} \in \mathscr{M}_{12}$.

For any $A_{21} \in \mathscr{M}_{21}$, by the relations $P_{1}\left(P_{1}+A_{21}\right)=P_{1}$, $\left(I-A_{21}\right)\left(P_{1}+A_{21}\right)=P_{1}$, and $\left(I+A_{21}\right)\left(P_{1}-A_{21}\right)=P_{1}$, and by using a similar argument to that of the above, one can show that

$$
-\delta\left(\xi A_{21}\right)=P_{1} \delta\left(A_{21}\right)-\xi \delta\left(A_{21}\right) P_{1}-\xi A_{21} \delta\left(P_{1}\right),
$$

and the claim also holds for $A_{21}$.

Claim 4. $L$ is an additive derivation with $L(\xi A)=\xi L(A)$ for each $A \in \mathscr{M}$.

We will prove the claim by considering three cases.

Case 1. Consider $\xi \neq 0,-1$. In this case, for any $A_{i j} \in \mathscr{M}_{i j}$, $1 \leq i \neq j \leq 2$, by Claim 3(1), (60), and (70), we get

$$
\delta\left(\xi A_{i j}\right)=\xi \delta\left(A_{i j}\right)+\xi \delta\left(P_{i}\right) A_{i j} .
$$

We will complete the proof of Claim 5 by the following several steps.

Step 1. For any $A_{i i} \in \mathscr{M}_{i i}, B_{j j} \in \mathscr{M}_{j j}, A_{i j}, B_{i j} \in \mathscr{M}_{i j}, 1 \leq$ $i \neq j \leq 2$, the following statements hold:

(i) $\delta\left(\xi A_{i i} B_{i j}\right)=\xi \delta\left(A_{i i}\right) B_{i j}+\xi A_{i i} \delta\left(B_{i j}\right)$; 
(ii) $\delta\left(\xi A_{i j} B_{j j}\right)=\xi \delta\left(A_{i j}\right) B_{j j}+\xi A_{i j} \delta\left(B_{j j}\right)$;

(iii) $\delta\left(A_{i i} B_{i j}\right)=\delta\left(A_{i i}\right) B_{i j}+A_{i i} \delta\left(B_{i j}\right)-\delta(I) A_{i i} B_{i j}$;

(iv) $\delta\left(A_{i j} B_{j j}\right)=\delta\left(A_{i j}\right) B_{j j}+A_{i j} \delta\left(B_{j j}\right)-\delta(I) A_{i j} B_{j j}$.

For any $A_{11} \in \mathscr{M}_{11}$, that is, invertible, and any $B_{12} \in \mathscr{M}_{12}$, since $\left(A_{11}^{-1}+B_{12}\right) A_{11}=P_{1}$, by Claim 2, Claim 3(1), and (58), we get $\delta\left(\xi A_{11} B_{12}\right)=\xi \delta\left(A_{11}\right) B_{12}+\xi A_{11} \delta\left(B_{12}\right)$. Now for any $A_{11} \in \mathscr{M}_{11}$, note that there exists some $c$ such that $c P_{1}-A_{11}$ is invertible in $\mathscr{M}_{11}$. It is easy to check that

$$
\begin{array}{r}
\delta\left(\xi A_{11} B_{12}\right)=\xi \delta\left(A_{11}\right) B_{12}+\xi A_{11} \delta\left(B_{12}\right) \\
\text { holds } \forall A_{11} \in \mathscr{M}_{11}, B_{12} \in \mathscr{M}_{12} .
\end{array}
$$

Also note that, by (71), $\delta\left(\xi A_{11} B_{12}\right)=\xi \delta\left(A_{11} B_{12}\right)+$ $\xi \delta\left(P_{1}\right) A_{11} B_{12}$. As $\xi \neq 0$, this and (72) yield

$$
\delta\left(A_{11} B_{12}\right)=\delta\left(A_{11}\right) B_{12}+A_{11} \delta\left(B_{12}\right)-\delta\left(P_{1}\right) A_{11} B_{12} .
$$

For any $B_{11} \in \mathscr{M}_{11}$ and $A_{21} \in \mathscr{M}_{21}$, one can similarly check that $\delta\left(\xi A_{21} B_{11}\right)=\xi \delta\left(A_{21}\right) B_{11}+\xi A_{21} \delta\left(B_{11}\right)$ and $\delta\left(A_{21} B_{11}\right)=\delta\left(A_{21}\right) B_{11}+A_{21} \delta\left(B_{11}\right)-A_{21} B_{11} \delta(I)$; for any $A_{22}, B_{22} \in \mathscr{M}_{22}, A_{12} \in \mathscr{M}_{12}$, and $B_{21} \in \mathscr{M}_{21}$, since $\left(P_{1}+\right.$ $\left.A_{12}\right)\left(P_{1}+B_{22}-A_{12} B_{22}\right)=P_{1}$ and $\left(P_{1}+A_{22}-A_{22} B_{21}\right)\left(P_{1}+\right.$ $\left.A_{21}\right)=P_{1}$, by the definition of $\delta$, Claim 3(1), (55), and (71), one can check that Step 1 holds.

Step 2. For any $A_{i i} \in \mathscr{M}_{i i}$, we have $\delta\left(\xi A_{i i} B_{i i}\right)=\xi \delta\left(A_{i i}\right) B_{i i}+$ $\xi A_{i i} \delta\left(B_{i i}\right), i=1,2$.

In fact, let $i \neq j$. For any $A_{i i}, B_{i i} \in \mathscr{M}_{i i}$ and any $S_{i j} \in \mathscr{M}_{i j}$, by (71) and Step 1, on the one hand, we have

$$
\begin{aligned}
\delta\left(\xi A_{i i} B_{i i} S_{i j}\right) & =\xi \delta\left(A_{i i} B_{i i} S_{i j}\right)+\xi \delta\left(P_{i}\right) A_{i i} B_{i i} S_{i j} \\
& =\xi \delta\left(A_{i i} B_{i i}\right) S_{i j}+\xi A_{i i} B_{i i} \delta\left(S_{i j}\right)
\end{aligned}
$$

on the other hand,

$$
\begin{aligned}
\delta( & \left.\xi A_{i i} B_{i i} S_{i j}\right) \\
& =\xi \delta\left(A_{i i} B_{i i} S_{i j}\right)+\xi \delta\left(P_{i}\right) A_{i i} B_{i i} S_{i j} \\
& =\xi \delta\left(A_{i i}\right) B_{i i} S_{i j}+\xi A_{i i} \delta\left(B_{i i} S_{i j}\right) \\
& =\xi \delta\left(A_{i i}\right) B_{i i} S_{i j}+\xi A_{i i} \delta\left(\xi \xi^{-1} B_{i i} S_{i j}\right) \\
& =\xi \delta\left(A_{i i}\right) B_{i i} S_{i j}+\xi^{2} A_{i i} \delta\left(\xi^{-1} B_{i i}\right) S_{i j}+\xi A_{i i} B_{i i} \delta\left(S_{i j}\right) .
\end{aligned}
$$

The above two equations yield that $\left(\delta\left(A_{i i} B_{i i}\right)-\delta\left(A_{i i}\right) B_{i i}-\right.$ $\left.\xi A_{i i} \delta\left(\xi^{-1} B_{i i}\right)\right) S_{i j}=0$ holds for all $A_{i i}, B_{i i} \in \mathscr{M}_{i i}$ and all $S_{i j} \in \mathscr{M}_{i j}$, which implies that $\left(\delta\left(\xi A_{i i} B_{i i}\right)-\xi \delta\left(A_{i i}\right) B_{i i}-\right.$ $\left.\xi A_{i i} \delta\left(B_{i i}\right)\right) S P_{j}=0$ holds for all $S \in \mathscr{M}$. It follows that $\delta\left(\xi A_{i i} B_{i i}\right)=\xi \delta\left(A_{i i}\right) B_{i i}+\xi A_{i i} \delta\left(B_{i i}\right)$ as $\overline{P_{j}}=I$. Step 2 is true.

Step 3. Consider $\delta(I)=L(I)=0$ and $\delta(\xi A)=\xi \delta(A)$ for all $A \in \mathscr{M}$.

Taking $A_{11}=B_{11}=P_{1}$ in Step 2, one obtains $\delta\left(\xi P_{1}\right)=2 \xi \delta\left(P_{1}\right)$, which and (55) yield $\delta\left(P_{1}\right)=0$. Note that
$\delta\left(P_{2}\right) A_{21}=A_{21} \delta\left(P_{1}\right)$ for each $A_{21}$ (Claim 3). It follows that $\delta\left(P_{2}\right) A_{21}=\delta\left(P_{2}\right) P_{2} A P_{1}=0$, which implies $\delta\left(P_{2}\right)=0$. So $\delta(I)=\delta\left(P_{1}\right)+\delta\left(P_{2}\right)=0$. Now it is obvious that $\delta(\xi A)=\xi \delta(A)$ for all $A \in \mathscr{M}$ by (71) and Step 2 .

Step 4. For any $A_{i i}, B_{i i} \in \mathscr{M}_{i i}$, we have $\delta\left(A_{i i} B_{i i}\right)=\delta\left(A_{i i}\right) B_{i i}+$ $A_{i i} \delta\left(B_{i i}\right), i=1,2$.

Let $i \neq j$. Take any $A_{i i}, B_{i i} \in \mathscr{M}_{i i}$ and any $S_{i j} \in \mathscr{M}_{i j}$. By Step 1, one gets

$$
\begin{gathered}
\delta\left(A_{i i} B_{i i} S_{i j}\right)=\delta\left(A_{i i} B_{i i}\right) S_{i j}+A_{i i} B_{i i} \delta\left(S_{i j}\right) \\
\delta\left(A_{i i} B_{i i} S_{i j}\right)= \\
=\delta\left(A_{i i}\right) B_{i i} S_{i j}+A_{i i} \delta\left(B_{i i} S_{i j}\right) \\
=\delta\left(A_{i i}\right) B_{i i} S_{i j}+A_{i i} \delta\left(B_{i i}\right) S_{i j}+A_{i i} B_{i i} \delta\left(S_{i j}\right) .
\end{gathered}
$$

Comparing the above two equations, and by Claims 2 and 4, one obtains that

$$
\left(\delta\left(A_{i i} B_{i i}\right)-\delta\left(A_{i i}\right) B_{i i}-A_{i i} \delta\left(B_{i i}\right)\right) S P_{j}=0
$$

holds for all $S \in \mathscr{M}$. It follows from the fact $\bar{P}_{j}=I$ that $\delta\left(A_{i i} B_{i i}\right)=\delta\left(A_{i i}\right) B_{i i}+A_{i i} \delta\left(B_{i i}\right)$.

Step 5. For any $A_{i j} \in \mathscr{M}_{i j}$ and $B_{j i} \in \mathscr{M}_{j i}$, we have $\delta\left(A_{i j} B_{j i}\right)=$ $\delta\left(A_{i j}\right) B_{j i}+A_{i j} \delta\left(B_{j i}\right), 1 \leq i \neq j \leq 2$.

Take any $A_{i j} \in \mathscr{M}_{i j}$ and $B_{j i} \in \mathscr{M}_{j i}$. Since $\left(P_{1}+A_{12}-\right.$ $\left.A_{12} B_{21}\right)\left(P_{1}+B_{21}\right)=P_{1}$, by Claim 3(1), Steps 1 and 3, we have

$$
\begin{aligned}
\xi \delta\left(B_{21} A_{12}\right)+\delta\left(A_{12}\right) B_{21}-\delta\left(A_{12} B_{21}\right) \\
\quad-\xi B_{21} \delta\left(A_{12}\right)+A_{12} \delta\left(B_{21}\right)-\xi \delta\left(B_{21}\right) A_{12}=0
\end{aligned}
$$

It follows that $\delta\left(A_{12} B_{21}\right)=\delta\left(A_{12}\right) B_{21}+A_{12} \delta\left(B_{21}\right)$ and $\delta\left(B_{21} A_{12}\right)=\delta\left(B_{21}\right) A_{12}+B_{21} \delta\left(A_{12}\right)$ as $\xi \neq 0$.

Step 6. For any $A, B \in \mathscr{M}$, we have $\delta(A B)=\delta(A) B+A \delta(B)$. Furthermore, $L$ is an additive derivation.

For any $A=A_{11}+A_{12}+A_{21}+A_{22}, B=B_{11}+B_{12}+$ $B_{21}+B_{22} \in \mathscr{M}$, by the additivity of $\delta$, and Steps $1-5$, one can easily check that $\delta(A B)=\delta(A) B+A \delta(B)$. Note that $L(A)=$ $\delta(A)-S A+A S$ for all $A \in \mathscr{M}$. So $L$ is an additive derivation.

Case 2. Consider $\xi=-1$. In this case, by (55), we get $\delta\left(P_{1}\right)=$ 0 . Then (58) implies

$$
\left[\delta\left(A_{11}^{-1}\right), A_{11}\right]_{-1}+\left[A_{11}^{-1}, \delta\left(A_{11}\right)\right]_{-1}=0
$$

for all invertible $A_{11} \in \mathscr{M}_{11}$.

Step 1. For any $A_{12} \in \mathscr{M}_{12}, A_{21} \in \mathscr{M}_{12}$ and $A_{22} \in \mathscr{M}_{22}$, we have $\delta\left(A_{12} A_{22}\right)=\delta\left(A_{12}\right) A_{22}+A_{12} \delta\left(A_{22}\right)+A_{22} \delta\left(A_{12}\right)$ and $\delta\left(A_{22} A_{21}\right)=\delta\left(A_{22}\right) A_{21}+A_{22} \delta\left(A_{21}\right)+\delta\left(A_{21}\right) A_{22}$. 
Take any $A_{12} \in \mathscr{M}_{12}$ and $A_{22} \in \mathscr{M}_{22}$. Since $\left(P_{1}+A_{12}\right)\left(P_{1}+\right.$ $\left.A_{22}-A_{12} A_{22}\right)=P_{1}$, by Claim 2 and Claim 3(2), one can verify

$$
\begin{aligned}
\delta\left(A_{12}\right)= & \delta\left(\left[P_{1}+A_{12}, P_{1}+A_{22}-A_{12} A_{22}\right]_{-1}\right) \\
= & {\left[\delta\left(A_{12}\right), P_{1}+A_{22}-A_{12} A_{22}\right]_{-1} } \\
& +\left[P_{1}+A_{12}, \delta\left(A_{22}\right)-\delta\left(A_{12} A_{22}\right)\right]_{-1} \\
= & \delta\left(A_{12}\right) P_{1}+\delta\left(A_{12}\right) A_{22} \\
& -\delta\left(A_{12}\right) A_{12} A_{22}+P_{1} \delta\left(A_{12}\right) \\
& +A_{22} \delta\left(A_{12}\right) \\
& -A_{12} A_{22} \delta\left(A_{12}\right)-P_{1} \delta\left(A_{12} A_{22}\right) \\
& +A_{12} \delta\left(A_{22}\right)-A_{12} \delta\left(A_{12} A_{22}\right) \\
& -\delta\left(A_{12} A_{22}\right) P_{1}-\delta\left(A_{12} A_{22}\right) A_{12} .
\end{aligned}
$$

Multiplying by $P_{1}$ and $P_{2}$ from the left and the right sides, respectively, in (80), and by Claim 3(2), we have

$$
\begin{aligned}
P_{1} \delta\left(A_{12} A_{22}\right) P_{2} & =P_{1} \delta\left(A_{12}\right) A_{22}+A_{12} \delta\left(A_{22}\right) \\
& =\delta\left(A_{12}\right) A_{22}+A_{12} \delta\left(A_{22}\right) ;
\end{aligned}
$$

multiplying by $P_{2}$ and $P_{1}$ from the left and the right sides, respectively, in (80), and by Claim 3(2) again, we have

$$
P_{2} \delta\left(A_{12} A_{22}\right) P_{1}=A_{22} \delta\left(A_{12}\right) P_{1}=A_{22} \delta\left(A_{12}\right) .
$$

So

$$
\begin{aligned}
\delta\left(A_{12} A_{22}\right) & =P_{1} \delta\left(A_{12} A_{22}\right) P_{2}+P_{2} \delta\left(A_{12} A_{22}\right) P_{1} \\
& =\delta\left(A_{12}\right) A_{22}+A_{12} \delta\left(A_{22}\right)+A_{22} \delta\left(A_{12}\right) .
\end{aligned}
$$

For any $A_{21} \in \mathscr{M}_{12}$ and $A_{22} \in \mathscr{M}_{22}$, by the same argument as above for the equation $\left(P_{1}+A_{22}-A_{22} A_{21}\right)\left(P_{1}+A_{21}\right)=$ $P_{1}$, one can check that

$$
\delta\left(A_{22} A_{21}\right)=\delta\left(A_{22}\right) A_{21}+A_{22} \delta\left(A_{21}\right)+\delta\left(A_{21}\right) A_{22} .
$$

Step 2. For any $A_{21} \in \mathscr{M}_{21}, A_{12} \in \mathscr{M}_{12}$, and $A_{11} \in \mathscr{M}_{11}$, we have $\delta\left(A_{11} A_{12}\right)=\delta\left(A_{11}\right) A_{12}+A_{11} \delta\left(A_{12}\right)+\delta\left(A_{12}\right) A_{11}$ and $\delta\left(A_{21} A_{11}\right)=\delta\left(A_{21}\right) A_{11}+A_{21} \delta\left(A_{11}\right)+A_{11} \delta\left(A_{21}\right)$.

For any invertible $A_{11} \in \mathscr{M}_{11}$ and any $A_{12} \in \mathscr{M}_{12}$, since $\left(A_{11}^{-1}+A_{12}\right) A_{11}=P_{1}$, by Claim 2 and (79), one obtains

$$
\begin{aligned}
\delta\left(A_{11} A_{12}\right)= & \delta\left(\left[A_{11}^{-1}+A_{12}, A_{11}\right]_{-1}\right) \\
= & {\left[\delta\left(A_{11}^{-1}\right)+\delta\left(A_{12}\right), A_{11}\right]_{-1} } \\
& +\left[A_{11}^{-1}+A_{12}, \delta\left(A_{11}\right)\right]_{-1} \\
= & \delta\left(A_{11}\right) A_{12}+A_{11} \delta\left(A_{12}\right)+\delta\left(A_{12}\right) A_{11} .
\end{aligned}
$$

Now it is easy to check that

$$
\begin{array}{r}
\delta\left(A_{11} A_{12}\right)=\delta\left(A_{11}\right) A_{12}+A_{11} \delta\left(A_{12}\right)+\delta\left(A_{12}\right) A_{11} \\
\text { holds } \forall A_{11} \in \mathscr{M}_{11}, A_{12} \in \mathscr{M}_{12} .
\end{array}
$$

For any invertible $A_{11} \in \mathscr{M}_{11}$ and any $A_{21} \in \mathscr{M}_{21}$, as $A_{11}$ $\left(A_{21}+A_{11}^{-1}\right)=P_{1}$, by a similar argument to that of the above, one can check that

$$
\delta\left(A_{21} A_{11}\right)=\delta\left(A_{21}\right) A_{11}+A_{21} \delta\left(A_{11}\right)+A_{11} \delta\left(A_{21}\right) .
$$

Step 3. For any $A_{i i} \in \mathscr{M}_{i i}$, we have $\delta\left(A_{i i}^{2}\right)=\delta\left(A_{i i}\right) A_{i i}+A_{i i} \delta$ $\left(A_{i i}\right), i=1,2$.

Let $1 \leq i \neq j \leq 2$. Then, for any $A_{i i} \in \mathscr{M}_{i i}$ and $S_{i j} \in \mathscr{M}_{i j}$, calculating $\delta\left(A_{i i} A_{i i} S_{i j}\right)$ by two different ways, one can easily check that Step 3 is true.

Step 4. For any $A_{12} \in \mathscr{M}_{12}$ and $A_{21} \in \mathscr{M}_{21}$, we have $\delta\left(A_{12} A_{21}\right)=\delta\left(A_{12}\right) A_{21}+A_{12} \delta\left(A_{21}\right)$ and $\delta\left(A_{21} A_{12}\right)=$ $\delta\left(A_{21}\right) A_{12}+A_{21} \delta\left(A_{12}\right)$.

In fact, since $\left(P_{1}+A_{12}-A_{12} A_{21}\right)\left(P_{1}+A_{21}\right)=P_{1}$, by Claim 2, Claim 3(2), and Step 2, one can verify

$$
\begin{aligned}
& \delta\left(A_{12}\right)-\delta\left(A_{12} A_{21}\right)+\delta\left(A_{21}\right)+\delta\left(A_{21} A_{12}\right) \\
&=\delta\left(\left[P_{1}+A_{12}-A_{12} A_{21}, P_{1}+A_{21}\right]_{-1}\right) \\
&= {\left[\delta\left(A_{12}\right)-\delta\left(A_{12} A_{21}\right), P_{1}+A_{21}\right]_{-1} } \\
&+\left[P_{1}+A_{12}-A_{12} A_{21}, \delta\left(A_{21}\right)\right]_{-1} \\
&= \delta\left(A_{12}\right) P_{1}+\delta\left(A_{12}\right) A_{21} \\
&-\delta\left(A_{12} A_{21}\right)+P_{1} \delta\left(A_{12}\right)-\delta\left(A_{12} A_{21}\right) \\
&+A_{21} \delta\left(A_{12}\right)+P_{1} \delta\left(A_{21}\right)+A_{12} \delta\left(A_{21}\right) \\
&+\delta\left(A_{21}\right) P_{1}+\delta\left(A_{21}\right) A_{12} .
\end{aligned}
$$

Multiplying by $P_{i}(i=1,2)$ from both sides in the above equation, one obtains

$$
\begin{aligned}
\delta\left(A_{12} A_{21}\right) & =P_{1} \delta\left(A_{12}\right) A_{21}+A_{12} \delta\left(A_{21}\right) P_{1} \\
& =\delta\left(A_{12}\right) A_{21}+A_{12} \delta\left(A_{21}\right), \\
\delta\left(A_{21} A_{12}\right) & =P_{2} \delta\left(A_{21}\right) A_{12}+A_{21} \delta\left(A_{12}\right) P_{2} \\
& =\delta\left(A_{21}\right) A_{12}+A_{21} \delta\left(A_{12}\right) .
\end{aligned}
$$

Step 5. For any $A \in \mathscr{M}$, we have $\delta\left(A^{2}\right)=\delta(A) A+A \delta(A)$. Furthermore, $L$ is an additive derivation.

For any $A=A_{11}+A_{12}+A_{21}+A_{22} \in \mathscr{M}$, by the additivity of $\delta$ and Steps 1-4, it is easy to verify $\delta\left(A^{2}\right)=\delta(A) A+A \delta(A)$. Note that $L(A)=\delta(A)+A S-S A$ for each $A$. So $L$ is an additive Jordan derivation. Now by [18], $L$ is an additive derivation 
because von Neumann algebras are semiprime and 2-torsion free.

Case 3. Consider $\xi=0$. In this case, $\delta$ in fact satisfies $A B=$ $P \Rightarrow \delta(A) B+A \delta(B)=\delta(P)$. So, by (55), $\delta\left(P_{1}\right)=0$. Then, (58) yields

$$
\delta\left(A_{11}\right) A_{11}^{-1}+A_{11} \delta\left(A_{11}^{-1}\right)=0
$$

Step 1. For any $A_{12} \in \mathscr{M}_{12}, B_{21} \in \mathscr{M}_{21}$, and $A_{22}, B_{22} \in \mathscr{M}_{22}$, we have $\delta\left(A_{12} B_{22}\right)=\delta\left(A_{12}\right) B_{22}+A_{12} \delta\left(B_{22}\right)$ and $\delta\left(A_{22} B_{21}\right)=$ $\delta\left(A_{22}\right) B_{21}+A_{22} \delta\left(B_{21}\right)$.

Note that $\left(P_{1}+A_{12}\right)\left(P_{1}+B_{22}+A_{12} B_{22}\right)=P_{1}$ and $\left(P_{1}+\right.$ $\left.A_{22}-A_{22} B_{21}\right)\left(P_{1}+B_{21}\right)=P_{1}$. By Claim 3(1), it is easy to check that Step 1 is true.

Step 2. For any $A_{22}, B_{22} \in M_{22}$, we have $\delta\left(A_{22} B_{22}\right)=$ $\delta\left(A_{22}\right) B_{22}+A_{22} \delta\left(B_{22}\right)$.

Take any $A_{22}, B_{22} \in \mathscr{M}_{22}$ and any $S_{21}$. By using Step 1 and calculating $\delta\left(A_{22} B_{22} S_{21}\right)$ by two different ways, Step 2 is easily checked.

Note that Step 2 implies $\delta\left(P_{2}\right)=0$.

Step 3. For any $A_{11}, B_{11} \in \mathscr{M}_{11}, A_{21} \in \mathscr{M}_{21}$, and $B_{12} \in \mathscr{M}_{12}$, we have $\delta\left(A_{11} B_{12}\right)=\delta\left(A_{11}\right) B_{12}+A_{11} \delta\left(B_{12}\right)$ and $\delta\left(A_{21} B_{11}\right)=$ $\delta\left(A_{21}\right) B_{11}+A_{21} \delta\left(B_{11}\right)$.

First, for any invertible $A_{11}, B_{11} \in \mathscr{M}_{11}$ and any $A_{21} \in$ $\mathscr{M}_{21}, B_{12} \in \mathscr{M}_{12}$, since $\left(A_{11}-A_{11} B_{12}\right)\left(A_{11}^{-1}+B_{12}+P_{2}\right)=P_{1}$ and $\left(B_{11}^{-1}+A_{21}+P_{2}\right)\left(B_{11}-A_{21} B_{11}\right)=P_{1}$, by Claim 2, Claim $3(1)$, the fact $\delta\left(P_{2}\right)=0$, and (91), one can obtain that Step 3 holds. Note that, for any $A_{11} \in \mathscr{M}_{11}$, there exists a scalar $c$ such that $c P_{1}-A_{11}$ is invertible in $\mathscr{M}_{11}$. It is easy to check that Step 3 also holds for all $A_{11}$ and $B_{11} \in \mathscr{M}_{11}$.

Step 4. For any $A_{11}, B_{11} \in \mathscr{M}_{11}$, we have $\delta\left(A_{11} B_{11}\right)=$ $\delta\left(A_{11}\right) B_{11}+A_{11} \delta\left(B_{11}\right)$.

Take any $A_{11}, B_{11} \in \mathscr{M}_{11}$ and any $S_{12} \in \mathscr{M}_{12}$. By Step 3, calculating $\delta\left(A_{11} B_{11} S_{12}\right)$ by two different ways, Step 4 is true.

Step 5. For any $A_{12} \in \mathscr{M}_{12}$ and $B_{21} \in \mathscr{M}_{21}$, we have $\delta\left(A_{12} B_{21}\right)=\delta\left(A_{12}\right) B_{21}+A_{12} \delta\left(B_{21}\right)$ and $\delta\left(B_{21} A_{12}\right)=$ $B_{21} \delta\left(A_{12}\right)+\delta\left(B_{21}\right) A_{12}$.

For any $A_{12} \in \mathscr{M}_{12}$ and $B_{21} \in \mathscr{M}_{21}$, since $\left(P_{1}+A_{12}-\right.$ $\left.A_{12} B_{21}\right)\left(P_{1}+B_{21}\right)=P_{1}$, by Claim 2, Claim 3(1), and the fact $\delta\left(P_{1}\right)=0$, we get $\delta\left(A_{12} B_{21}\right)=\delta\left(A_{12}\right) B_{21}+A_{12} \delta\left(B_{21}\right)$. Thus, by Steps 1 and 3 , for any $C_{12} \in \mathscr{M}$, we have

$$
\begin{aligned}
\delta( & \left.B_{21} A_{12}\right) C_{21}+B_{21} A_{12} \delta\left(C_{21}\right) \\
& =\delta\left(B_{21} A_{12} C_{21}\right) \\
& =\delta\left(B_{21}\right) A_{12} C_{21}+B_{21} \delta\left(A_{12} C_{21}\right) \\
& =\delta\left(B_{21}\right) A_{12} C_{21}+B_{21} \delta\left(A_{12}\right) C_{21}+B_{21} A_{12} \delta\left(C_{21}\right)
\end{aligned}
$$

which implies $\left(\delta\left(B_{21} A_{12}\right)-\delta\left(B_{21}\right) A_{12}-B_{21} \delta\left(A_{12}\right)\right) C P_{1}=0$ for all $C \in \mathscr{M}$. It follows from the fact $\bar{P}_{1}=I$ that $\delta\left(B_{21} A_{12}\right)=$ $\delta\left(B_{21}\right) A_{12}+B_{21} \delta\left(A_{12}\right)$. So Step 5 is true.
Now by Steps 1-5 and similar arguments as that in the proof of Theorem 1, one can check that $\delta$ is a derivation. As $L(A)=\delta(A)-S A+A S$ for each $A, L$ is also a derivation.

The proof of the theorem is finished.

By Theorem 4, we can give different characterizations of additive $\xi$-Lie derivations on von Neumann algebras.

Corollary 5. Let $\mathscr{M}$ be a von Neumann algebra without central summands of type $I_{1}$. Suppose that $L: \mathscr{M} \rightarrow \mathscr{M}$ is an additive map and $\xi$ is a scalar with $\xi \neq 1$. Then, the following statements are equivalent.

(1) $L$ is a $\xi$-Lie derivation.

(2) $L$ is a derivation and $L(\xi A)=\xi L(A)$ for each $A \in \mathscr{M}$.

(3) $L$ satisfies $L\left([A, B]_{\xi}\right)=[L(A), B]_{\xi}+[A, L(B)]_{\xi}$ for any $A, B \in \mathscr{M}$ with $A B=P$, where $P$ is some fixed projection with $\underline{P}=0$ and $\bar{P}=I$.

\section{Conflict of Interests}

The authors declare that there is no conflict of interests regarding the publication of this paper.

\section{Acknowledgments}

The authors wish to express their thanks to the referees for their helpful comments and suggestions. This work is partially supported by the National Natural Science Foundation of China (11101250) and Youth Foundation of Shanxi Province (2012021004).

\section{References}

[1] J. A. Brooke, P. Busch, and D. B. Pearson, "Commutativity up to a factor of bounded operators in complex Hilbert space," The Royal Society of London A, vol. 458, no. 2017, pp. 109-118, 2002.

[2] C. Kassel, Quantum Groups, vol. 155, Springer, New York, NY, USA, 1995.

[3] X. Qi and J. Hou, "Additive Lie ( $\xi$-Lie) derivations and generalized Lie ( $\xi$-Lie) derivations on nest algebras," Linear Algebra and its Applications, vol. 431, no. 5-7, pp. 843-854, 2009.

[4] M. Y. Huang and J. H. Zhang, " $\xi$-Lie derivable maps on triangular algebras by Jordan zero products," Acta Mathematica Sinica, vol. 55, no. 5, pp. 953-960, 2012.

[5] W. Yang and J. Zhu, "Characterizations of additive (generalized) $\xi$-Lie $(\alpha, \beta)$ derivations on triangular algebras," Linear and Multilinear Algebra, vol. 61, no. 6, pp. 811-830, 2013.

[6] M. Brešar, "Characterizing homomorphisms, derivations and multipliers in rings with idempotents," Proceedings of the Royal Society of Edinburgh A, vol. 137, no. 1, pp. 9-21, 2007.

[7] J. Hou and X. Qi, "Additive maps derivable at some points on $J$-subspace lattice algebras," Linear Algebra and its Applications, vol. 429, no. 8-9, pp. 1851-1863, 2008.

[8] T.-K. Lee, "Generalized skew derivations characterized by acting on zero products," Pacific Journal of Mathematics, vol. 216, no. 2, pp. 293-301, 2004.

[9] Z. Pan, "Derivable maps and derivational points," Linear Algebra and its Applications, vol. 436, no. 11, pp. 4251-4260, 2012. 
[10] X. Qi and J. Hou, "Linear maps Lie derivable at zero on Jsubspace lattice algebras," Studia Mathematica, vol. 197, no. 2, pp. 157-169, 2010.

[11] X. Qi, J. Cui, and J. Hou, "Characterizing additive $\xi$-Lie derivations of prime algebras by $\xi$-Lie zero products," Linear Algebra and its Applications, vol. 434, no. 3, pp. 669-682, 2011.

[12] X. F. Qi, J. Ji, and J. Hou, "Characterizationof additive maps $\xi$ Lie derivable at zero on von Neumann Algebras," submitted. In press.

[13] F. Lu and W. Jing, "Characterizations of Lie derivations of $B(X)$," Linear Algebra and its Applications, vol. 432, no. 1, pp. 89-99, 2010.

[14] P. Ji and W. Qi, "Characterizations of Lie derivations of triangular algebras," Linear Algebra and its Applications, vol. 435, no. 5, pp. 1137-1146, 2011.

[15] X. Qi and J. Hou, "Characterization of Lie derivations on prime rings," Communications in Algebra, vol. 39, no. 10, pp. 38243835, 2011.

[16] X. Qi and J. Hou, "Characterization of Lie derivations on von Neumann algebras," Linear Algebra and its Applications, vol. 438, no. 1, pp. 533-548, 2013.

[17] C. R. Miers, "Lie homomorphisms of operator algebras," Pacific Journal of Mathematics, vol. 38, pp. 717-735, 1971.

[18] M. Brešar, "Jordan derivations on semiprime rings," Proceedings of the American Mathematical Society, vol. 104, no. 4, pp. 10031006, 1988. 


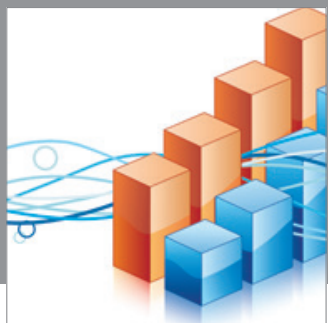

Advances in

Operations Research

mansans

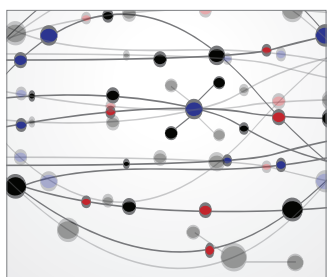

The Scientific World Journal
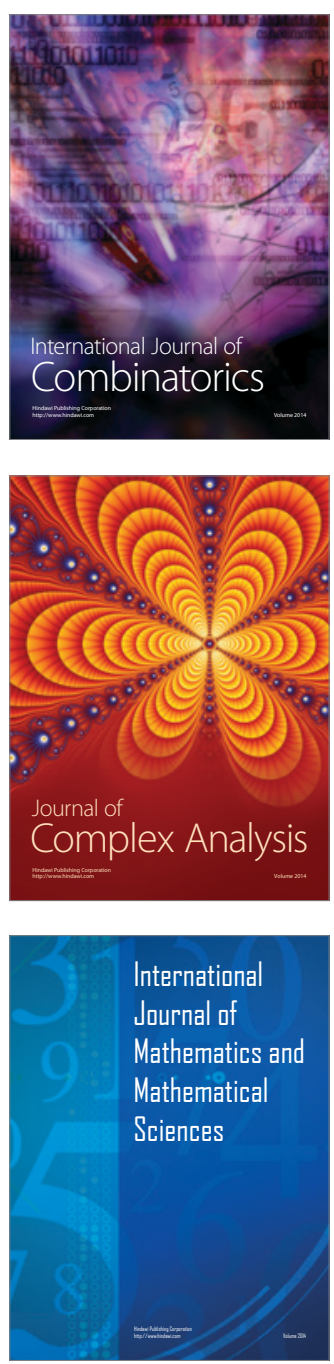
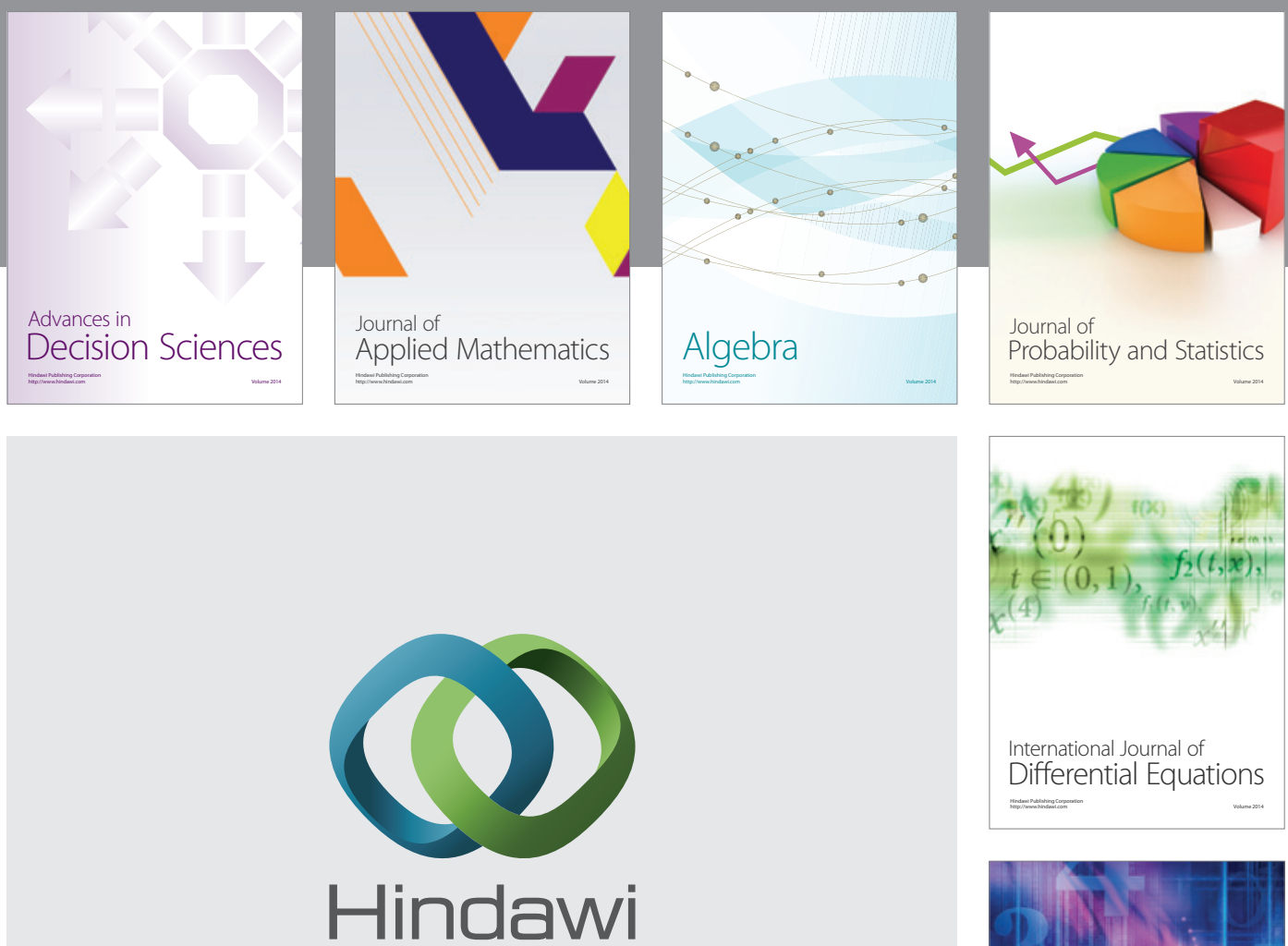

Submit your manuscripts at http://www.hindawi.com
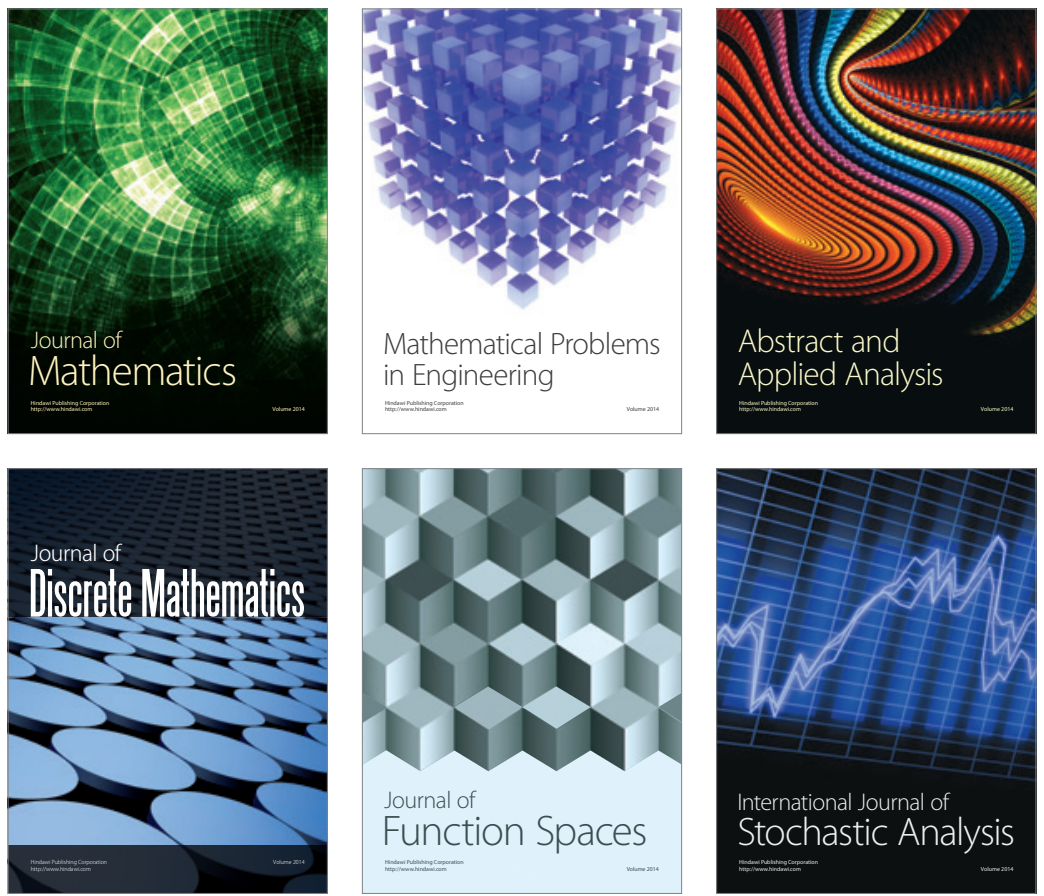

Journal of

Function Spaces

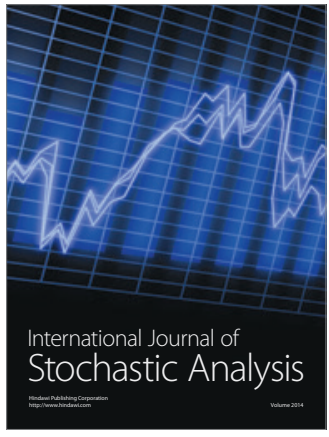

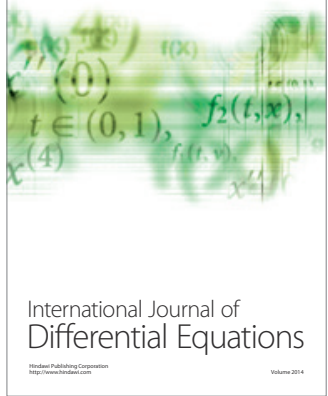
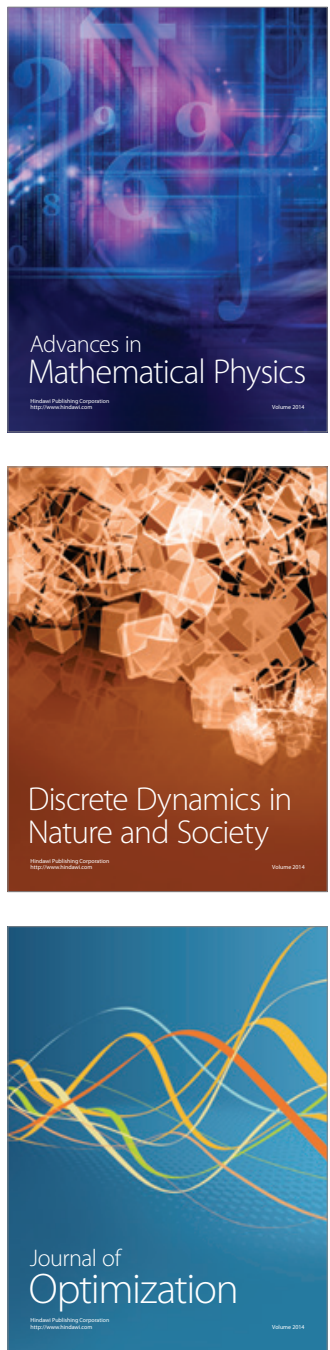